\title{
An Inventory Model for Deteriorating Items under Conditionally Permissible Delay in Payments Depending on the Order Quantity
}

\author{
Sumana Bera1', Samarjit Kar², Tripti Chakraborti³ ${ }^{3}$ Bani Kumar Sinha ${ }^{4}$ \\ ${ }^{1}$ Department of Basic Science and Humanities, Future Institute of Engineering \& Management, Kolkata, India \\ ${ }^{2}$ Department of Mathematics, National Institute of Technology Durgapur, Durgapur, India \\ ${ }^{3}$ Department of Applied Mathematics, University of Calcutta, Kolkata, India \\ ${ }^{4}$ Department of Operations, Supply Chain and Retail Management, Calcutta Business School, Kolkata, India \\ Email: kars k@yahoo.com
}

Received 25 July 2014; revised 19 August 2014; accepted 10 September 2014

Copyright (C) 2014 by authors and Scientific Research Publishing Inc.

This work is licensed under the Creative Commons Attribution International License (CC BY).

http://creativecommons.org/licenses/by/4.0/

(c) (i) Open Access

\section{Abstract}

The purpose of this inventory model is to investigate the retailer's optimal replenishment policy under permissible delay in payments. In this paper, we assume that the supplier would offer the retailer partially permissible delay in payments when the order quantity is smaller than a predetermined quantity (W). The most inventory systems are usually formed without considering the effect of deterioration of items which deteriorate continuously like fresh fruits, vegetables etc. Here we consider the loss due to deterioration. In real world situation, the demand of some items varies with change of seasons and occasions. So it is more significant if the loss of deterioration is time dependent. Considering all these facts, this inventory model has been developed to make more realistic and flexible marketing policy to the retailer, also establish the result by ANOVA analysis by treating different model parameters as factors.

\section{Keywords}

Inventory, Economic Order Quantity (EOQ), Deterioration, Permissible Delay in Payment, Weibull Distribution

\section{Introduction}

The general, economic order quantity (EOQ) model assumes that the retailer must be paid for the items as soon as the items are received. However, in practical situation, the supplier offers to the retailer many incentives such 
as a cash discount to motivate faster payment and stimulate sales, or a permissible delay in payments to attract new customers and increase sales. Before the end of the permissible delay period, the retailer can sell the goods and accumulate revenue and earn interest. On the other hand, a higher interest is charged if the payment is not settled by the end of the trade credit period. Therefore, it makes economic sense for the retailer to delay the settlement of the replenishment account up to the last moment of the permissible period allowed by the supplier.

Moreover, the most inventory systems are usually formed without considering the effect of deterioration. In real-life situations there are products such as volatile, liquids, some medicines, food materials, etc., in which the rate of deterioration is very large with time. Therefore, the loss due to deterioration should not be neglected. So in this model, we are considering the items such as fresh fruits and vegetables which have the exponential distribution for the time to deterioration.

Several authors discussing this topic have appeared in the literatures that investigate inventory problems under varying conditions. Some of the papers are discussed below. Goyal [1] developed an EOQ model under the conditions of permissible delay in payments. Aggarwal and Jaggi [2] extended Goyal's [1] model to consider the deteriorating items. Chang, Ouyang and Teng [3] then established an EOQ model for deteriorating items under supplier's trade credits linked to order quantity. Chung and Liao [4] studied a similar lot-sizing problem under supplier's trade credits depending on the retailer's order quantity.

However, most of the papers dealing with EOQ in the presence of permissible delay in payments assume that the supplier only offers the retailer fully permissible delay in payments if the retailer orders a sufficient quantity. Otherwise, permissible delay in payments would not be permitted. We know that this policy of the supplier to stimulate the demands from the retailer is very practical. But this is just an extreme case. That is, the retailer would obtain $100 \%$ permissible delay in payments if the retailer ordered a large enough quantity. Otherwise, $0 \%$ permissible delay in payments would happen.

Huang [5] established an EOQ model in which the supplier offers a partially permissible delay in payments when the order quantity is smaller than the prefixed quantity $\mathrm{W}$. In the above paper, a partially permissible delay in payments means the retailer must make a partial payment to the supplier when the order is received to enjoy some portion of the trade credit. Then, the retailer must pay off the remaining balances at the end of the permissible delay period. For example, the supplier provides $100 \%$ delay payment permitted if the retailer orderes a predetermined quantity, otherwise only $\lambda \%(0 \leq \lambda \leq 100)$ delay payment permitted. From the viewpoint of supplier's marketing policy, the supplier can use the fraction of the permissible delay in payments to attract and stimulate the demands from the retailer. Ouyang [6] studied the similar EOQ model with constant deterioration of the quantity. Das et al. [7] presented an EPQ model for deteriorating items under permissible delay in payment. Teng et al. [8] developed an EOQ model for stock dependent demand to supplier's trade credit with a progressive payment scheme. Min et al. [9] developed an EPQ model with inventory-level dependent demand and permissible delay in payment. Recently, Ouyang and Chang [10] proposed an optimal production lot with imperfect production process under permission delay in payment and complete backlogging.

The present EOQ model based on the fact that the suppliers would offer a partially permissible delay in payment if the retailer ordered more than or equal to a predetermined quantity $\mathrm{W}$. If the ordering quantity is less than $\mathrm{W}$, then the retailer has to pay off a certain amount (which is decided by the supplier) at the ordering time. In the real-world situation, we generalize the inventory model by relaxing some facts as 1) the retailer's selling price per unit is higher than its purchase unit cost; 2) the interest rate charged by the bank is not necessarily higher than the retailer's investment return rate; 3 ) many items like as fresh fruits and vegetables deteriorate exponentially with time.

In this regard, we model a retailer's inventory system as a cost minimization problem to determine the retailer's optimal inventory cycle time and optimal order quantity. Several theorems are established to describe the optimal replenishment policy for the retailer under the more general framework and use an approach to solve this complex inventory problem. Finally, numerical example has been given to illustrate all these theorems and sensitivity analysis has been done. Also we have established the result by ANOVA analysis by treating different model parameters as factors.

\section{Mathematical Notations and Assumptions}

In this section, the present study develops a retailer's inventory model under conditionally permissible delay in payments. The following notation and assumptions are used throughout the paper. 


\subsection{Notation}

$D$ : the annual demand

$A$ : the ordering cost per order

$W$ : the quantity at which the fully delay payment permitted per order

$P$ : the purchasing cost per unit

$H$ : the unit holding cost per year excluding interest charge

$S$ : the selling price per unit

$I_{e}$ : the interest earned per dollar per year

$I_{k}$ : the interest charged per dollar in stock per year

$M$ : the period of permissible delay in settling accounts

$\lambda$ : the fraction of delay payment permitted by the supplier per order, $0 \leq \lambda \leq 1$

$Z(t)=\alpha \beta t^{\beta-1}$ : is two parameter Weibull distribution function representing time to deterioration, where $\alpha=$ scale parameter, $(0<\alpha \ll 1), \beta=$ shape parameter $(\beta>1)$

$T$ : the replenishment cycle time in years

$Q$ : the order quantity

$T C R(T)$ : the annual total relevant cost, which is a function of $T$

$T^{*}$ : the optimal replenishment cycle time of $T C R(T)$

$Q^{*}$ : the optimal order quantity $=D T^{*}$

\subsection{Assumptions}

1) Replenishments are instantaneous.

2) Demand rate, $D$, is known and constant.

3) Shortages are not allowed.

4) Inventory system involves only one type of inventory.

5) Time horizon is infinite.

\section{Mathematical Formulation}

The inventory level decreases due to demand as well as deterioration. Thus, the change of inventory level can be represented by the following differential equation:

$$
\frac{\mathrm{d} I(t)}{\mathrm{d} t}+Z(t) I(t)=-D, 0<t<T
$$

where $Z(t)=\alpha \beta t^{\beta-1}$, with boundary condition $\mathrm{I}(\mathrm{t})=0$. The solution of $(1)$ with $\mathrm{e}^{\alpha t^{\beta-1}}=1+\alpha t^{\beta} \quad$ (as $\left.\alpha \ll 1\right)$ is

$$
I(t)=D \mathrm{e}^{-\alpha t^{\beta}}\left[(T-t)+\left(\frac{\alpha}{\beta+1}\right)\left(T^{\beta+1}-t^{\beta+1}\right)\right] ; \quad 0 \leq t \leq T
$$

Hence, the order quantity for each cycle is

$$
Q=I(0)=D\left[T+\left(\frac{\alpha}{\beta+1}\right) T^{\beta+1}\right]
$$

From (3), we can obtain the time interval $T_{w}$ that $\mathrm{W}$ units are depleted to zero due to both demand and deterioration. We put $Q=W$ in (3) and get $T_{w}$ from (3)

$$
W=D\left[T_{w}+\left(\frac{\alpha}{\beta+1}\right) T_{w}^{\beta+1}\right]
$$

If $Q \geq W$ (i.e., $T \geq T_{w}$ ), the fully delayed payment is permitted, otherwise, partial delayed payment is permitted if $Q<W$ (i.e., $T<T_{w}$ ), the retailer must have to pay supplier, the partial payment of $(1-\lambda) p Q$ at time 0 . From the constant sales revenue $s D$, the retailer will be able to pay off the loan $(1-\lambda) p Q$ at time 


$$
(1-\lambda)(p / s)\left[T+\left(\frac{\alpha}{\beta+1}\right) T^{\beta+1}\right](=G(\text { say }))
$$

At time $T_{0}$, the pay of time $G$ of the partial payment is shorter or equal to the trade credit period M. i.e., $G \leq$ $M$.

Therefore we get $T_{0}$ from the following relation

$$
(1-\lambda)(p / s)\left[T_{0}+\left(\frac{\alpha}{\beta+1}\right) T_{0}^{\beta+1}\right] \equiv M
$$

It is obvious that always $M<T_{0}$ and if $T \leq T_{0}$ then $G \leq M$ and vice-versa.

Based on the values of $M, T_{w}, T_{0}$, we have three possible cases:

1) $T_{w} \leq M \leq T_{0}$, 2) $\left.M<T_{w} \leq T_{0}, 3\right) \quad M<T_{0}<T_{w}$

a) Annual ordering cost $=\frac{A}{T}$,

b) Annual stock holding cost excluding interest charge

$$
H=h / T \int_{0}^{T} I(t) \mathrm{d} t=h / T \int_{0}^{T} D \mathrm{e}^{-\alpha t^{\beta}}\left[(T-t)+\left(\frac{\alpha}{\beta+1}\right)\left(T^{\beta+1}-t^{\beta+1}\right)\right] \mathrm{d} t
$$

Simplifying with $\mathrm{e}^{-\alpha t^{\beta}}=1-\alpha t^{\beta} \quad$ (as $\left.\alpha \ll 1\right)$

$$
H=D h T\left[\frac{1}{2}+\frac{\alpha \beta T^{\beta}}{(\beta+1)(\beta+2)}-\frac{\alpha^{2} T^{2(\beta+1)}}{2(\beta+1)^{2}}\right]
$$

$H=D h T\left[\frac{1}{2}+\frac{\alpha \beta T^{\beta}}{(\beta+1)(\beta+2)}\right]$ (Neglecting $\alpha^{2}$ term, since $\left.\alpha \ll 1\right)$

c) Annual deteriorating cost $=\frac{1}{T}[p Q-p D T]=D p\left(\frac{\alpha}{\beta+1}\right) T^{\beta}$

\subsection{Case 1: $T_{w} \leq M<T_{0}$}

There are three sub-cases in terms of annual opportunity cost of the capital which are depicted in Figure 1.

\subsubsection{Sub-Case 1.1: $M \leq T$}

The retailer starts paying the interest for the items in stock after time $M$ with rate $I_{k}$ and during time 0 to $M$, from the sale revenue the retailer earns the interest with rate $I_{e}$, therefore in this sub-case, the annual opportunity cost of capital is

Inventory level [I(t)]

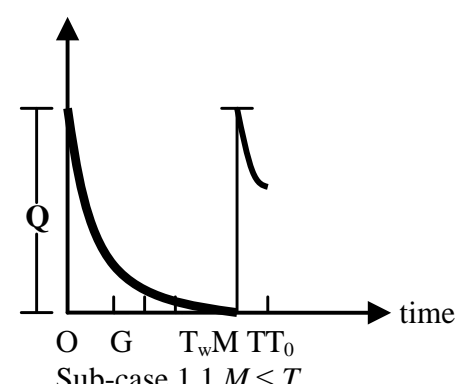

Inventory level [I(t)]

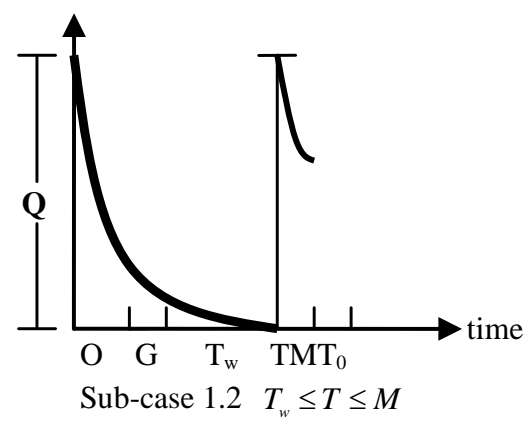

Inventory level [I(t)]

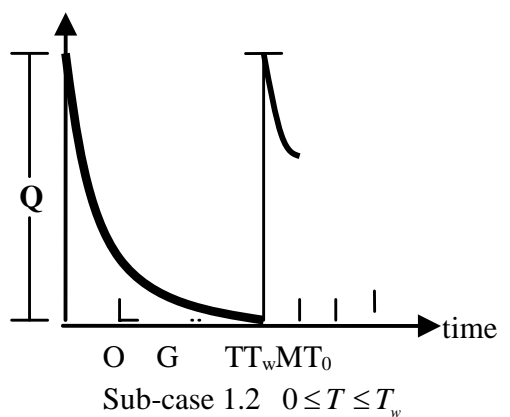

Figure 1. Graphical representation of three different situations of case 1. 


$$
\frac{p I_{k} D}{T}\left[\frac{T^{2}}{2}+\frac{M^{2}}{2}-T M+\frac{\alpha \beta}{(\beta+1)(\beta+2)}\left(T^{(\beta+2)}-M^{(\beta+2)}\right)+\frac{\alpha}{(\beta+1)}\left(M^{\beta}-T^{\beta}\right) T M\right]-\frac{s I_{e} D M^{2}}{2 T}
$$

3.1.2. Sub-Case 1.2: $T_{w} \leq T \leq M$

In this case, there is no interest paid for financing inventory, therefore in this sub-case, the annual opportunity cost of capital is $-s I_{e} D\left[M-\frac{T}{2}\right]$

\subsubsection{Sub-Case 1.3: $0<T<T_{w}$}

If $T<T_{w}$, then the retailer must have to pay the partial payment $(1-\lambda) p Q$ at time 0 to the supplier, and retailer pays off the loan to the bank from sales revenue at time

$$
G=(1-\lambda)(p / s)\left[T+\left(\frac{\alpha}{\beta+1}\right) T^{\beta+1}\right]
$$

Consequently, the interest is charged on the partial payment from time 0 to $G$. Hence the annual interest payable is

$$
\frac{I_{k}}{T} \int_{0}^{G}\{(1-\lambda) p Q-s D t\} \mathrm{d} t=\frac{I_{k} D}{2 T}\left\{(1-\lambda)^{2}\left(p^{2} / s\right)\left(T+\frac{\alpha}{\beta+1} T^{(\beta+1)}\right)^{2}\right\}
$$

Similarly, the interest earned starts from time $G$ to $M$, and the annual earned interest is

$$
\begin{aligned}
& \frac{I_{e}}{T}\left[\int_{G}^{T}\{s D t-(1-\lambda) p Q\} \mathrm{d} t+\int_{T}^{M}\{s D T-(1-\lambda) p Q\} \mathrm{d} t\right] \\
& =\frac{s D I_{e}}{2 T}\left[T-(1-\lambda)(p / s)\left(T+\frac{\alpha}{\beta+1} T^{(\beta+1)}\right)\right]^{2}+\frac{s D I_{e}}{T}(M-T)\left[T-(1-\lambda)(p / s)\left(T+\frac{\alpha}{\beta+1} T^{(\beta+1)}\right)\right]
\end{aligned}
$$

Therefore in this sub-case, the annual opportunity cost of capital is

$$
\begin{aligned}
& \frac{I_{k} D}{2 T}\left\{(1-\lambda)^{2}\left(p^{2} / s\right)\left(T+\frac{\alpha}{\beta+1} T^{(\beta+1)}\right)^{2}\right\}-\frac{s D I_{e}}{2 T}\left[T-(1-\lambda)(p / s)\left(T+\frac{\alpha}{\beta+1} T^{(\beta+1)}\right)\right]^{2} \\
& -\frac{s D I_{e}}{T}(M-T)\left[T-(1-\lambda)(p / s)\left(T+\frac{\alpha}{\beta+1} T^{(\beta+1)}\right)\right]
\end{aligned}
$$

Therefore in case 1 , the annual relevant cost can be expressed as

$$
\operatorname{TRC}(T)= \begin{cases}T R C_{1}(T), & M \leq T \\ T R C_{2}(T), & T_{w} \leq T \leq M \\ T R C_{3}(T), & 0<T<T_{w}\end{cases}
$$

where,

$$
\begin{aligned}
& T R C_{1}(T)=\left\{\frac{A}{T}+D h T\left[\frac{1}{2}+\frac{\alpha \beta T^{\beta}}{(\beta+1)(\beta+2)}\right]+D p\left(\frac{\alpha}{\beta+1}\right) T^{\beta}+\frac{p I_{k} D}{T}\left[\frac{T^{2}}{2}+\frac{M^{2}}{2}-T M\right.\right. \\
&\left.\left.+\frac{\alpha \beta}{(\beta+1)(\beta+2)}\left(T^{(\beta+2)}-M^{(\beta+2)}\right)+\frac{\alpha}{(\beta+1)}\left(M^{\beta}-T^{\beta}\right) T M\right]-\frac{s I_{e} D M^{2}}{2 T}\right\} \\
& T_{R C}(T)=\frac{A}{T}+D h T\left[\frac{1}{2}+\frac{\alpha \beta T^{\beta}}{(\beta+1)(\beta+2)}\right]+D p\left(\frac{\alpha}{\beta+1}\right) T^{\beta}-s I_{e} D\left[M-\frac{T}{2}\right]
\end{aligned}
$$




$$
\begin{aligned}
T R C_{3}(T)= & \left\{\frac{A}{T}+D h T\left[\frac{1}{2}+\frac{\alpha \beta T^{\beta}}{(\beta+1)(\beta+2)}\right]+D p\left(\frac{\alpha}{\beta+1}\right) T^{\beta}\right. \\
& +\frac{I_{k} D}{2 T}\left[(1-\lambda)^{2}\left(\frac{p^{2}}{s}\right)\left(T+\frac{\alpha}{\beta+1} T^{(\beta+1)}\right)^{2}\right] \\
& -\frac{s I_{e} D}{2 T}\left[T-(1-\lambda)\left(\frac{p}{s}\right)\left(T+\frac{\alpha}{\beta+1} T^{(\beta+1)}\right)\right]^{2} \\
& \left.-\frac{s I_{e} D}{2 T}(M-T)\left[T-(1-\lambda)\left(\frac{p}{s}\right)\left(T+\frac{\alpha}{\beta+1} T^{(\beta+1)}\right)\right]\right\}
\end{aligned}
$$

\subsection{Case 2: $M<T_{w} \leq T_{0}$ (cf. Figure 2)}

Similar to case 1, three different sub-cases are as follows:

\subsubsection{Sub-Case 2.1: $T_{w} \leq T$}

This case is similar to the sub-case 1.1 (where $M \leq T$ ). Since $M<T_{w} \leq T$, therefore the total relevant cost is same as $T R C_{1}(T)$.

\subsubsection{Sub-Case 2.2: $M \leq T<T_{w}$}

Since $T<T_{w}$, the retailer must have to pay the partial payment $(1-\lambda) p Q$ at time 0 to the supplier, and retailer pays off the loan to the bank from sales revenue at time $G$. The annual interest payable from 0 to time $G$ is given by

$$
\frac{I_{k}}{T} \int_{0}^{G}[(1-\lambda) p Q-s D t] \mathrm{d} t=\frac{I_{k} D}{2 T}\left[(1-\lambda)^{2}\left(\frac{p^{2}}{s}\right)\left(T+\frac{\alpha}{\beta+1} T^{(\beta+1)}\right)^{2}\right]
$$

Again since $M \leq T$, the retailer has to pay interest from time $M$ to time $T$. Therefore, the annual payable interest is

$$
\frac{p I_{k}}{T} \int_{M}^{T} I(t) \mathrm{d} t=\frac{p I_{k} D}{T}\left[\frac{T^{2}}{2}+\frac{M^{2}}{2}-T M+\frac{\alpha \beta}{(\beta+1)(\beta+2)}\left(T^{(\beta+2)}-M^{(\beta+2)}\right)+\frac{\alpha}{(\beta+1)}\left(M^{\beta}-T^{\beta}\right) T M\right]
$$

Similarly, the interest earned starts from time $G$ to $M$, and thus the annual interest earned is

$$
\frac{I_{e}}{T} \int_{G}^{M}[s D t-(1-\lambda) p Q] \mathrm{d} t=\frac{s I_{e} D}{2 T}\left[M-(1-\lambda)\left(\frac{p}{s}\right)\left(T+\frac{\alpha}{(\beta+1)} T^{\beta+1}\right)\right]^{2}
$$

In this sub-case, the annual relevant cost is

Inventory level [I(t)]

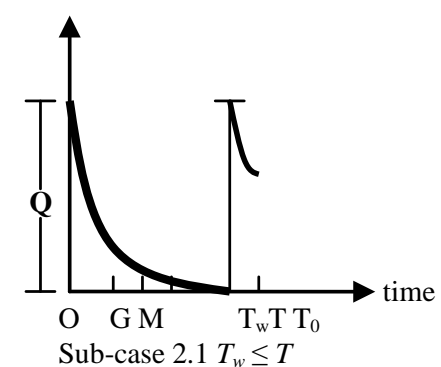

Inventory level [I(t)]

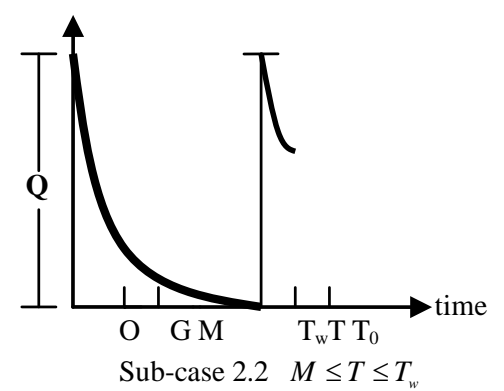

Inventory level [I(t)]

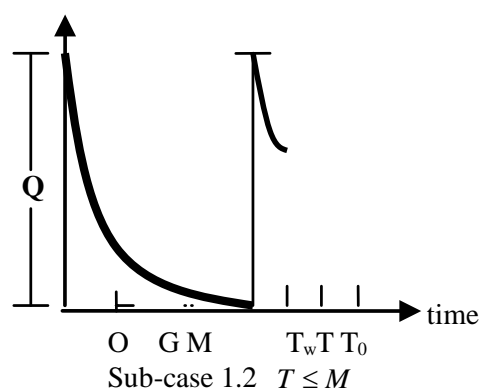

Figure 2. Graphical representation of three different situations of Case 2. 


$$
\begin{aligned}
\operatorname{TRC}_{4}(T)= & \frac{A}{T}+D h t\left[\frac{1}{2}+\frac{\alpha \beta T^{\beta}}{(\beta+1)(\beta+2)}\right]+D p \frac{\alpha}{(\beta+1)} T^{\beta}+\frac{I_{k} D}{2 T}\left[(1-\lambda)^{2}\left(\frac{p^{2}}{s}\right)\left(T+\frac{\alpha}{(\beta+1)} T^{\beta+1}\right)^{2}\right] \\
& +\frac{p I_{k} D}{T}\left[\frac{T^{2}}{2}+\frac{M^{2}}{2}-T M+\frac{\alpha \beta}{(\beta+1)(\beta+2)}\left(T^{\beta+2}-M^{\beta+2}\right)+\frac{\alpha}{(\beta+1)}\left(M^{\beta}-T^{\beta}\right) T M\right] \\
& -\frac{s D I_{e}}{2 T}\left[M-(1-\lambda)\left(\frac{p}{s}\right)\left(T+\frac{\alpha}{(\beta+1)} T^{\beta+1}\right)\right]^{2}
\end{aligned}
$$

\subsubsection{Sub-Case 2.3: $T \leq M$}

This sub-case is similar to the Sub-Case 1.3 (where $T<T_{w}<M$ ). Since $T \leq M<T_{w}$, the annual total relevant cost is same as $T C R_{3}(T)$.

Therefore in Case 2 the annual relevant cost can be expressed as

$$
\operatorname{TRC}(T)= \begin{cases}T R C_{1}(T), & T_{w} \leq T \\ T R C_{4}(T), & M \leq T \leq T_{w} \\ T R C_{3}(T), & T<M\end{cases}
$$

\subsection{Case 3: $M<T_{0}<T_{w}$ (cf. Figure 3)}

There are four sub-cases as following:

\subsubsection{Sub-Case $3.1 T_{w} \leq T$}

This case is similar to the Sub-Case 1.1 (where $M \leq T$ ). Since $M<T_{0}<T_{w} \leq T$, therefore the total relevant cost is $T R C_{1}(T)$.

3.3.2. Sub-Case $3.2 T_{0} \leq T<T_{w}$

If $T_{0} \leq T$, then $M<G$, i.e. $M<(1-\lambda)\left(\frac{p}{s}\right)\left[T+\frac{\alpha}{\beta+1} T^{\beta+1}\right]$.

Inventory level [I(t)]

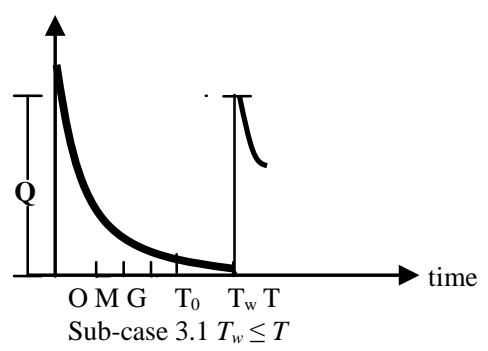

Inventory level [I(t)]

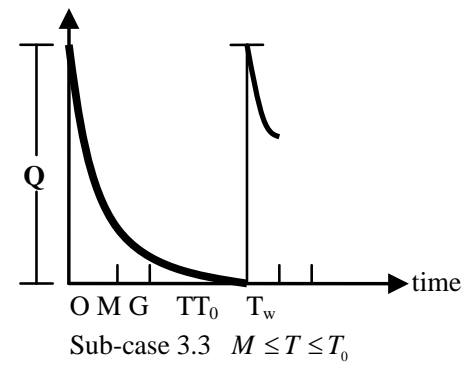

Inventory level [I(t)]

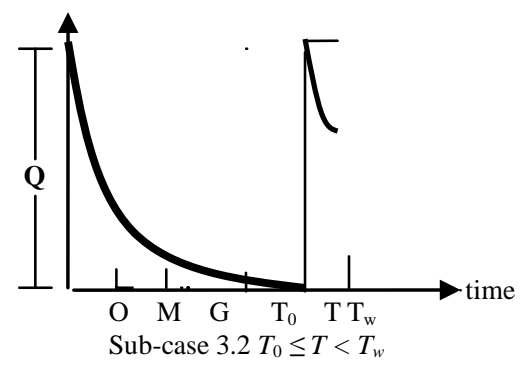

Inventory level [I(t)]

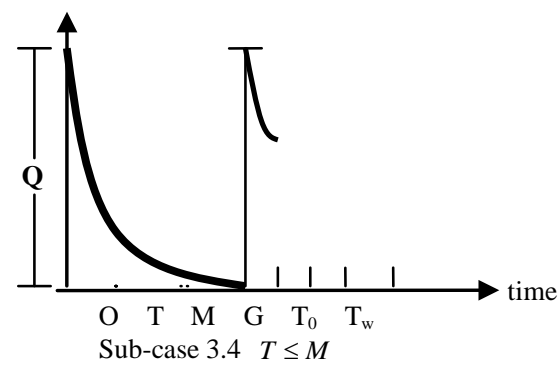

Figure 3. Graphical representation of four different situations of Case 3. 
In this sub-case, at the beginning i.e. at time 0 , the retailer must take a loan to pay the supplier the partial payment of $(1-\lambda) p Q$.

Since $M<G$, the retailer have to take another loan to pay the rest of $\lambda p Q$ at time $M$.

Again since the first loan will be paid from the sale revenue until $t=(1-\lambda)\left(\frac{p}{s}\right)\left[T+\frac{\alpha}{\beta+1} T^{\beta+1}\right]$.

Hence, the retailer gets the second loan at time $M$ but can start paying off from the sales revenue after time $t=$ $G(>M)$. As a result, there is no interest earned, but have to pay the interest. The $1^{\text {st }}$ payable interest is

$$
I_{k} \int_{0}^{G}[(1-\lambda) p Q-s D t] \mathrm{d} t=\frac{I_{k} D}{2}\left[(1-\lambda)^{2}\left(\frac{p^{2}}{s}\right)\left(T+\frac{\alpha}{\beta+1} T^{\beta+1}\right)^{2}\right] .
$$

For the $2^{\text {nd }}$ loan $\lambda p Q$, retailer has to pay interest at $I_{k}$ rate per year from $M$ to $G$. Therefore, the $2^{\text {nd }}$ payable interest is

$$
I_{k} \int_{M}^{G} \lambda p Q \mathrm{~d} t=I_{k} \lambda p D\left[T+\frac{\alpha}{\beta+1} T^{\beta+1}\right] \cdot(G-M)=I_{k} \lambda p D\left[(1-\lambda)\left(\frac{p}{s}\right)\left(T+\frac{\alpha}{\beta+1} T^{\beta+1}\right)-M\right]
$$

Again, since the retailer has started to pay off the loan $\lambda p Q$ from the sales revenue after time $G$ the loan will be completely paid off at time $\frac{\lambda p Q}{s D}$.

Therefore, the $3^{\text {rd }}$ payable interest is $I_{k} \int_{0}^{\frac{\lambda p Q}{s D}}(\lambda p Q-s D t) \mathrm{d} t=\frac{I_{k} Q}{2}\left[\lambda^{2}\left(\frac{p^{2}}{s}\right)\left(T+\frac{\alpha}{\beta+1} T^{\beta+1}\right)\right]$

Therefore the annual total payable interest is

$$
\begin{aligned}
& \frac{I_{k} D}{2 T}\left[(1-\lambda)^{2}\left(\frac{p^{2}}{s}\right)\left(T+\frac{\alpha}{\beta+1} T^{\beta+1}\right)^{2}\right]+\frac{I_{k} \lambda p D}{T}\left[(1-\lambda)\left(\frac{p}{s}\right)\left(T+\frac{\alpha}{\beta+1} T^{\beta+1}\right)-M\right] \\
& +\frac{I_{k} Q}{2 T}\left[\lambda^{2}\left(\frac{p^{2}}{s}\right)\left(T+\frac{\alpha}{\beta+1} T^{\beta+1}\right)\right]
\end{aligned}
$$

\subsubsection{Sub-Case $3.3 M \leq T \leq T_{0}$}

This case is similar to the Sub-Case 2.2 (where $M \leq T<T_{w}$ ). Since $M \leq T \leq T_{0}<T_{w}$, the annual total relevant cost is $T R C_{4}(T)$.

\subsubsection{Sub-Case 3.4 $T \leq M$}

This case is similar to the Sub-Case 1.3 (where $0<T<T_{w}$ ).

Since $T \leq M<T_{0}<T_{w}$, therefore the annual total relevant cost is $T R C_{3}(T)$.

In Case 3 , the annual relevant cost can be expressed as

where,

$$
\operatorname{TRC}(T)= \begin{cases}T R C_{1}(T), & T_{w} \leq T \\ T R C_{5}(T), & T_{0} \leq T \leq T_{w} \\ T R C_{4}(T), & M \leq T<T_{0} \\ T R C_{3}(T), & T \leq M\end{cases}
$$

$$
\begin{aligned}
\operatorname{TRC}_{5}(T)= & \frac{A}{T}+D h T\left[\frac{1}{2}+\frac{\alpha \beta T^{\beta}}{(\beta+1)(\beta+2)}\right]+D p \frac{\alpha}{\beta+1} T^{\beta}+\frac{I_{k} D}{2 T}\left[\left(1-2 \lambda+2 \lambda^{2}\right)\left(\frac{p^{2}}{s}\right)\left(T+\frac{\alpha}{\beta+1} T^{\beta+1}\right)^{2}\right] \\
& +\frac{I_{k} \lambda p Q}{T}\left[(1-\lambda)\left(\frac{p}{s}\right)\left(T+\frac{\alpha}{\beta+1} T^{\beta+1}\right)-M\right]
\end{aligned}
$$




\section{Theoretical Results}

Now we try to determine the optimal replenishment cycle time $(T)$ that minimizes the annual relevant cost.

\subsection{Case $1 T_{w} \leq M<T_{0}$}

To minimize $T R C_{1}(T)$ in (9) for $M \leq T$ we get $\frac{{\mathrm{d} T R C_{1}(T)}_{\mathrm{d} T}=0}{2}$

$$
\begin{aligned}
& \Rightarrow D h\left[\frac{T^{2}}{2}+\frac{\alpha \beta T^{\beta+2}}{\beta+2}\right]+D p\left[\frac{\alpha \beta}{\beta+1}\right] T^{\beta+1} \\
& +p I_{k} D\left[\frac{T^{2}-M^{2}}{2}+\frac{\alpha \beta}{(\beta+2)} T^{\beta+2}+\frac{\alpha \beta}{(\beta+1)(\beta+2)} M^{\beta+2}-\frac{\alpha \beta}{(\beta+1)} M T^{\beta+1}\right] \\
& +\frac{s I_{e} D M^{2}}{2}-A=0
\end{aligned}
$$

Now there exists a value $T$ in $[M, \infty)$ at which we get minimum value of $T R C_{1}(T)$. Let

$$
\left.\Delta_{1} \equiv \frac{\mathrm{d} T R C_{1}(T)}{\mathrm{d} T}\right|_{T=M} \Rightarrow \Delta_{1} \equiv D h\left[\frac{M^{2}}{2}+\frac{\alpha \beta M^{\beta+2}}{\beta+2}\right]+D p \frac{\alpha \beta M^{\beta+1}}{\beta+1}+\frac{s I_{e} D M^{2}}{2}-A
$$

Then we have the following lemma.

\section{Lemma 1:}

a) If $\Delta_{1} \leq 0$, then the annual total relevant cost $T R C_{1}(T)$ has the unique minimum value at the point $T=T_{1}$, where $T_{1} \in[M, \infty)$ and satisfies (17).

b) If $\Delta_{1}>0$, the annual total relevant cost $T R C_{1}(T)$ has a minimum value at the boundary point $T=M$.

Proof: Let

$$
\begin{aligned}
& F_{1}(T)= {\left[D h\left(\frac{1}{2} T^{2}+\frac{\alpha(\beta)}{\beta+2} T^{\beta+2}\right)+D p \frac{\alpha(\beta)}{\beta+1} T^{\beta+1}+p I_{k} D\left(\frac{1}{2}\left(T^{2}-M^{2}\right)+\frac{\alpha(\beta)}{\beta+2} T^{\beta+2}\right.\right.} \\
&\left.\left.+\frac{\alpha(\beta)}{(\beta+1)(\beta+2)} M^{\beta+2}-\frac{\alpha(\beta)}{(\beta+1)} M T^{\beta+1}\right)+\frac{1}{2} s I_{e} D M^{2}-A\right] \\
& T \in[M, \infty)
\end{aligned}
$$

Taking the derivative of $F_{1}(T)$ with respect to $T \in(M, \infty)$, we get

$$
\begin{aligned}
\frac{\mathrm{d} F_{1}(T)}{\mathrm{d} T} & =D h\left[T+\alpha(\beta) T^{\beta+1}\right]+D p\left(\alpha(\beta) T^{\beta}\right)+p I_{k} D\left[T+\alpha(\beta) T^{\beta+1}-\alpha(\beta) M T^{\beta}\right] \\
& =D h\left[T+\alpha(\beta) T^{\beta+1}\right]+D p\left(\alpha(\beta) T^{\beta}\right)+p I_{k} D\left[T+\alpha(\beta) T^{\beta}(T-M)\right]>0
\end{aligned}
$$

Therefore, $F_{1}(T)$ is strictly increasing function of $T$ in $[M, \infty)$. From (A1), we get

$$
F_{1}(M)=\Delta_{1} \leq 0 \text {, and } \lim _{T \rightarrow \infty} F_{1}(T)=+\infty
$$

Therefore, if $F_{1}(M)=\Delta_{1} \leq 0$, then by applying the Darboux's theorem†, $\exists$ a unique $T_{1} \in[M, \infty)$ such that $F_{1}\left(T_{1}\right)=0$. Again taking the second order derivative of $T R C_{1}(T)$ with respect to $T$ at $T_{1}$, we have

$$
\frac{\mathrm{d}^{2} T R C_{1}(T)}{\mathrm{d} T^{2}}=\frac{\mathrm{d}}{\mathrm{d} T}\left\{\frac{F_{1}(T)}{T^{2}}\right\}, \quad\left[\because \frac{\mathrm{d} T R C_{1}(T)}{\mathrm{d} T}=\frac{F_{1}(T)}{T^{2}}\right]=\frac{-2 F_{1}(T)}{T^{3}}+\frac{1}{T^{2}} \frac{\mathrm{d} F_{1}(T)}{\mathrm{d} T}
$$

Hence, 


$$
\left.\frac{\mathrm{d}^{2} T R C_{1}(T)}{\mathrm{d} T^{2}}\right|_{T=T_{1}}=\frac{1}{T^{2}}\left[D h\left\{T_{1}+\alpha(\beta) T_{1}^{\beta+1}\right\}+D p \alpha(\beta) T_{1}^{\beta}+p I_{k} D\left\{T_{1}+\alpha(\beta) T_{1}^{\beta}(T-M)\right\}\right]>0
$$

Therefore $T_{1} \in[M, \infty)$ is the unique minimum solution to $T R C_{1}(T)$.

On the other hand, if $F_{1}(M)=\Delta_{1}>0$, we have $F_{1}(T)>0, \forall T \in[M, \infty)$. Consequently, we know that

$$
\frac{\mathrm{d} T R C_{1}(T)}{\mathrm{d} T}=\frac{F_{1}(T)}{T^{2}}>0, \quad \forall T \in(M, \infty) .
$$

That is, $T R C_{1}(T)$ is a strictly increasing function of $T$ in $[M, \infty)$. Therefore, $T R C_{1}(T)$ has a minimum value at the boundary point $T=M$. This completes the proof.

\section{Darboux's Theorem:}

If a function $f$ is derivable on a closed in terval $[a, b]$ and $f^{\prime}(a), f(b)$ are of opposite signs then there exist at least one point $c \in[a, b]$ such that $f^{\prime}(c)=0$.

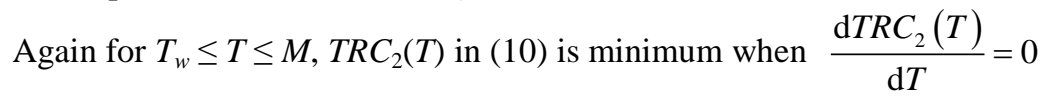

$$
\begin{aligned}
& D h\left[\frac{1}{2}+\frac{\alpha \beta}{\beta+2} T^{\beta}\right]+D p\left[\frac{\alpha \beta}{\beta+1}\right] T^{\beta-1}+\frac{s I_{e} D}{2}-\frac{A}{T^{2}}=0 \\
& D h\left[\frac{T^{2}}{2}+\frac{\alpha \beta}{\beta+2} T^{\beta+2}\right]+D p\left[\frac{\alpha \beta}{\beta+1}\right] T^{\beta+1}+\frac{s I_{e} D}{2} T^{2}-A=0
\end{aligned}
$$

To prove that there exist a value of $T$ in the interval $\left[T_{w}, M\right]$ at which minimizes $T R C_{2}(T)$, we let

$$
\begin{aligned}
& \left.\Delta_{2} \equiv \frac{\mathrm{d} T R C_{2}(T)}{\mathrm{d} T}\right|_{T=T_{W}} \\
& \Delta_{2} \equiv D h\left[\frac{T_{W}^{2}}{2}+\frac{\alpha \beta}{\beta+2} T_{W}^{\beta+2}\right]+D p\left[\frac{\alpha \beta}{\beta+1}\right] T_{W}^{\beta+1}+\frac{S I_{e} D}{2} T_{W}^{2}-A
\end{aligned}
$$

It is obvious that $\Delta_{1} \geq \Delta_{2}$ if $M \geq T_{w}$, then we have the following lemma:

\section{Lemma 2:}

a) If $\Delta_{2} \leq 0 \leq \Delta_{1}$, then the annual total relevant cost $T R C_{2}(T)$ has the unique minimum value at the point $T=T_{2}$, where $T_{2} \in\left[T_{w}, M\right]$ and (19) is satisfied by $T_{2}$.

b) If $\Delta_{2}>0$, the annual total relevant cost $T R C_{2}(T)$ has a minimum value at the lower boundary point $T=T_{w}$.

c) If $\Delta_{1}<0$, the annual total relevant cost $T R C_{2}(T)$ has a minimum value at the upper boundary point $T=M$.

Proof: The proof is similar to that in Lemma 1 so we omit it.

Similarly, for $0<T<T_{w}, T R C_{3}(T)$ in (11) is minimum when $\frac{\mathrm{d} T R C_{3}(T)}{\mathrm{d} T}=0$. That is,

$$
\begin{aligned}
& D h\left[\frac{T^{2}}{2}+\frac{\alpha \beta}{\beta+2} T^{\beta+2}\right]+D p \frac{\alpha \beta}{\beta+1} T^{\beta+1}+\frac{I_{k} D}{2} T^{2}(1-\lambda)^{2}\left(\frac{p^{2}}{s}\right)\left[1+2 \alpha\left(T^{\beta}\right)+\frac{\alpha^{2}(1+2 \beta)}{(\beta+1)^{2}} T^{2 \beta}\right] \\
& +\frac{s D I_{e}}{2} T^{2}\left[\left\{1-(1-\lambda)\left(\frac{p}{s}\right)\left(1+\frac{\alpha}{\beta+1} T^{\beta}\right)\right\}\left\{1+(1-\lambda)\left(\frac{p}{s}\right)\left(1+\frac{\alpha(1+2 \beta)}{\beta+1} T^{\beta}\right)\right\}\right. \\
& \left.+2(M-T)(1-\lambda)\left(\frac{p}{s}\right) \frac{\alpha \beta}{\beta+1} T^{\beta-1}\right]-A=0
\end{aligned}
$$

Again there exist a value of $T$ in the interval $\left(0, T_{w}\right)$ which minimizes $T R C_{3}(T)$, we let 


$$
\begin{aligned}
\Delta_{3} \equiv & \left.\frac{\mathrm{d} T R C_{3}(T)}{\mathrm{d} T}\right|_{T=T_{W}} \text { or } \\
\Delta_{3} \equiv & \left\{D h\left(\frac{1}{2} T_{W}^{2}+\frac{\alpha \beta}{\beta+2} T_{W}^{\beta+2}\right)+D p \frac{\alpha \beta}{\beta+1} T_{W}^{\beta+1}+\frac{I_{k} D}{2} T_{W}^{2}(1-\lambda)^{2}\left(\frac{p^{2}}{s}\right)\left(1+2 \alpha\left(T_{W}^{\beta}\right)+\frac{\alpha^{2}(1+2 \beta)}{(\beta+1)^{2}} T_{W}^{2 \beta}\right)\right. \\
& +\frac{s D I_{e}}{2} T_{W}^{2}\left\{\left[1-(1-\lambda)\left(\frac{p}{s}\right)\left(1+\frac{\alpha}{\beta+1} T_{W}^{\beta}\right)\right]\left[1+(1-\lambda)\left(\frac{p}{s}\right)\left(1+\frac{\alpha(1+2 \beta)}{\beta+1} T_{W}^{\beta}\right)\right]\right. \\
& \left.\left.+2\left(M-T_{W}\right)(1-\lambda)\left(\frac{p}{s}\right) \frac{\alpha \beta}{\beta+1} T_{W}^{\beta-1}\right\}-A\right\}=0
\end{aligned}
$$

Then we have the following lemma:

\section{Lemma 3:}

a) If $\Delta_{3} \geq 0$, then the annual total relevant cost $T R C_{3}(T)$ has the unique minimum value at the point $T=T_{3}$, where $T_{3} \in\left(0, T_{w}\right)$ and satisfies (21).

b) If $\Delta_{3}<0$, then the value of $T \in\left(0, T_{w}\right)$ which minimizes $T R C_{3}(T)$ does not exist.

\section{Proof:}

Let

$$
\begin{aligned}
F_{3}(T)= & D h\left[\frac{T^{2}}{2}+\frac{\alpha \beta}{\beta+2} T^{\beta+2}\right]+D p \frac{\alpha \beta}{\beta+1} T^{\beta+1}+\frac{I_{k} D}{2} T^{2}(1-\lambda)^{2}\left(\frac{p^{2}}{s}\right)\left[1+2 \alpha\left(T^{\beta}\right)+\frac{\alpha^{2}(1+2 \beta)}{(\beta+1)^{2}} T^{2 \beta}\right] \\
& +\frac{s D I_{e}}{2} T^{2}\left[\left\{1-(1-\lambda)\left(\frac{p}{s}\right)\left(1+\frac{\alpha}{\beta+1} T^{\beta}\right)\right\}\left\{1+(1-\lambda)\left(\frac{p}{s}\right)\left(1+\frac{\alpha(1+2 \beta)}{\beta+1} T^{\beta}\right)\right\}\right. \\
& \left.+2(M-T)(1-\lambda)\left(\frac{p}{s}\right) \frac{\alpha \beta}{\beta+1} T^{\beta-1}\right]-A=0
\end{aligned}
$$

Differentiating $F_{3}(T)$ with respect to $T \in\left(0, T_{w}\right)$, we have

$$
\begin{aligned}
\frac{\mathrm{d} F_{3}(T)}{\mathrm{d} T}= & {\left[D h\left[T+\alpha \beta T^{\beta+1}\right]+D p \alpha(\beta) T^{\beta}+D T\left(I_{k}-I_{e}\right)(1-\lambda)^{2}\left(p^{2} / s\right)\right.} \\
& \left.\times\left\{1+\alpha(\beta+2) T^{\beta}+\frac{\alpha^{2}(1+2 \beta)}{(\beta+1)^{2}} T^{2 \beta}\right\}+s D I_{e} T\left\{1+\alpha \beta(1-\lambda)(p / s) M T^{\beta-1}\right\}\right]
\end{aligned}
$$

Since

$$
\begin{aligned}
& h+\alpha \beta h T^{\beta}+p \alpha(\beta) T^{\beta-1}+\left(I_{k}-I_{e}\right)(1-\lambda)^{2}\left(p^{2} / s\right)\left\{1+\alpha(\beta+2) T^{\beta}+\frac{\alpha^{2}(1+2 \beta)}{(\beta+1)^{2}} T^{2 \beta}\right\} \\
& +s I_{e}\left\{1+\alpha \beta(1-\lambda)(p / s) M T^{\beta-1}\right\} \geq h+\left(I_{k}-I_{e}\right)(1-\lambda)^{2}\left(p^{2} / s\right)+s I_{e} \\
& =h+(1-\lambda)^{2}\left(p^{2} / s\right) I_{k}+\left\{s-(1-\lambda)^{2}\left(p^{2} / s\right)\right\} I_{e}>0 \\
& {\left[\because s-(1-\lambda)^{2}\left(p^{2} / s\right)>0 \text { for } s \geq p \text { and } 0 \leq \lambda \leq 1\right]}
\end{aligned}
$$

Therefore we have $\frac{\mathrm{d} F_{3}(T)}{\mathrm{d} T}>0 \Rightarrow F_{3}(T)$ is a strictly increasing function of $T$ in $\left(0, T_{w}\right)$. Now from (B1), we have

$$
\lim _{T \rightarrow 0} F_{3}(T)=-A<0 \text { and } \lim _{T \rightarrow T_{W}^{-}} F_{3}(T)=\Delta_{3} .
$$


Therefore, if $\lim _{T \rightarrow T_{W}^{-}} F_{3}(T)=\Delta_{3} \geq 0$, then applying Darboux's theorem, $\exists$ a unique $T_{3} \in\left(0, T_{W}\right)$ such that $F_{3}\left(T_{3}\right)=0$. Again, taking the second order derivative of $T R C_{3}(T)$ with respect to $T$ at $T_{3}$, we have

$$
\begin{aligned}
\left.\frac{\mathrm{d}^{2} T R C_{3}(T)}{\mathrm{d} T^{2}}\right|_{T=T_{3}}= & \frac{1}{T_{3}} D\left[h+\alpha h(\beta) T_{3}^{\beta}+p \alpha(\beta) T_{3}^{\beta-1}+\left(I_{k}-I_{e}\right)(1-\lambda)^{2}\left(p^{2} / s\right)\right. \\
& \left.\times\left\{1+\alpha(\beta+2) T_{3}^{\beta}+\frac{\alpha^{2}(1+2 \beta)}{\beta+1} T_{3}^{2 \beta}\right\}+s I_{e}\left\{1+\alpha(\beta)(1-\lambda)(p / s) M T_{3}^{\beta-1}\right\}\right]>0
\end{aligned}
$$

Therefore, $T_{3} \in\left(0, T_{W}\right)$ is the unique minimum solution to $T R C_{3}(T)$. Again if $\lim _{T \rightarrow T_{W}^{\bar{W}}} F_{3}(T)=\Delta_{3}<0$, then $F_{3}(T)<0 \quad \forall T \in\left(0, T_{W}\right)$. We have

$$
\frac{\mathrm{d} F_{3}(T)}{\mathrm{d} T}=\frac{F_{3}(T)}{T^{2}}<0 \quad \forall T \in\left(0, T_{W}\right) .
$$

Therefore, $T R C_{3}(T)$ is a strictly decreasing function of $T$ in $\left(0, T_{W}\right)$, but we cannot find the value of $T$ in the open interval $\left(0, T_{W}\right)$ which minimizes $T R C_{3}(T)$. This completes the proof.

For $0 \leq \lambda \leq 1$, it can be written that $\Delta_{2} \leq \Delta_{3}$. Again $\mathrm{T}_{\mathrm{w}} \leq \mathrm{M}$, we that $\Delta_{2} \leq \Delta_{1}$. Now combining Lemmas $1-3$ and including the fact that $T R C_{1}(M)=T R C_{2}(M)$, for Case-1 we can obtain a theoretical result to determine the optimal cycle time $T^{*}$ as:

\section{Theorem 1:}

For $T_{w} \leq M<T_{0}$, the optimal replenishment cycle time $T^{*}$, that minimizes the annual total relevant cost is given as:

\begin{tabular}{ccc}
\hline Condition & $\operatorname{TRC}\left(T^{*}\right)$ & $T^{*}$ \\
\hline$\Delta_{1} \leq 0$ and $\Delta_{3}<0$ & $T R C_{1}\left(T_{1}\right)$ & $T_{1}$ \\
$\Delta_{1} \leq 0$ and $\Delta_{3} \geq 0$ & $\operatorname{Min}\left\{T R C_{1}\left(T_{1}\right), T R C_{3}\left(T_{3}\right)\right\}$ & $T_{1}$ or $T_{3}$ \\
$\Delta_{1}>0, \Delta_{3} \geq 0$ and $\Delta_{2}<0$ & $\operatorname{Min}\left\{T R C_{2}\left(T_{2}\right), T R C_{3}\left(T_{3}\right)\right\}$ & $T_{2}$ or $T_{3}$ \\
$\Delta_{2} \geq 0$ & $\operatorname{Min}\left\{T R C_{2}\left(T_{w}\right), T R C_{3}\left(T_{3}\right)\right\}$ & $T_{w}$ or $T_{3}$ \\
$\Delta_{1}>0$ and $\Delta_{3}<0$ & $T R C_{2}\left(T_{2}\right)$ & $T_{2}$ \\
\hline
\end{tabular}

\subsection{Case $2 M<T_{w} \leq T_{0}$}

For $T_{w} \leq T$, similar approach used in Case 1, the 1st order condition for $T R C_{1}(T)$ of (9) is the same as (17), so there exist a unique value of $T$ in $\left[T_{w}, \infty\right)$ at which $T R C_{1}(T)$ is minimized.

Let $\left.\Delta_{4} \equiv \frac{\mathrm{d} T R C_{1}(T)}{\mathrm{d} T}\right|_{T=T_{W}}$, then

$$
\begin{aligned}
\Delta_{4} \equiv & \left\{D h\left[\frac{1}{2} T_{w}^{2}+\frac{\alpha \beta}{\beta+2} T_{w}^{\beta+2}\right]+D p \frac{\alpha \beta}{\beta+1} T_{w}^{\beta+1}+p I_{k} D\left[\frac{1}{2}\left(T_{w}^{2}-M^{2}\right)+\frac{\alpha \beta}{\beta+2} T_{w}^{\beta+2}\right.\right. \\
& \left.\left.+\frac{\alpha \beta}{(\beta+1)(\beta+2)} M^{\beta+2}-\frac{\alpha \beta}{(\beta+1)} M T_{w}^{\beta+1}\right]+\frac{s I_{e} D}{2} M^{2}-A\right\}
\end{aligned}
$$

We have the following lemma:

\section{Lemma 4:}

a) If $\Delta_{4} \leq 0$, then the annual total relevant cost $T R C_{1}(T)$ has the unique minimum value at the point $T=T_{1}$, where $T_{1} \in\left[T_{w}, \infty\right)$ and satisfies (17).

b) If $\Delta_{4}>0$, the annual total relevant cost $T R C_{1}(T)$ has a minimum value at the boundary point $T=T_{w}$. 
Proof: The proof is similar to that in Lemma 1 so we omit it.

Again for $M \leq T<T_{w}$, the total relevant cost $T R C_{4}(T)$ in (13) is minimum when

$$
\begin{aligned}
& \frac{\mathrm{d} T R C_{4}(T)}{\mathrm{d} T}=0 \\
& \Rightarrow\left\{D h\left[\frac{1}{2} T^{2}+\frac{\alpha \beta}{\beta+2} T^{\beta+2}\right]+D p \frac{\alpha \beta}{\beta+1} T^{\beta+1}+\frac{1}{2} I_{k} D T^{2}(1-\lambda)^{2}\left(\frac{p^{2}}{s}\right)\left(1+2 \alpha\left(T^{\beta}\right)+\frac{\alpha^{2}(1+2 \beta)}{(\beta+1)^{2}} T^{2 \beta}\right)\right. \\
& \quad+\frac{1}{2} p I_{k} D\left[T^{2}-M^{2}+2 \frac{\alpha \beta}{\beta+2} T^{\beta+2}+2 \frac{\alpha \beta}{(\beta+1)(\beta+2)} M^{\beta+2}-2 \frac{\alpha \beta}{\beta+1} M T^{\beta+1}\right] \\
& \left.\quad+\frac{1}{2} S I_{e} D\left[M-(1-\lambda)\left(\frac{p}{s}\right)\left(T+\frac{\alpha}{\beta+1} T^{\beta+1}\right)\right]\left[M+(1-\lambda)\left(\frac{p}{s}\right)\left(T+\frac{\alpha(1+2 \beta)}{\beta+1} T^{\beta+1}\right)\right]-A\right\}=0
\end{aligned}
$$

To prove that there exist a unique value of $T$ in $\left[M, T_{w}\right)$ at where $T R C_{4}(T)$ is minimum, we let

$$
\begin{aligned}
\left.\Delta_{5} \equiv \frac{{\mathrm{d} T R C_{4}}_{4}(T)}{\mathrm{d} T}\right|_{T=M} \text {, then } \\
\Delta_{5} \equiv\left\{D h\left[\frac{1}{2} M^{2}+\frac{\alpha \beta}{\beta+2} M^{\beta+2}\right]+D p \frac{\alpha \beta}{\beta+1} M^{\beta+1}\right. \\
+\frac{1}{2} I_{k} D M^{2}(1-\lambda)^{2}\left(\frac{p^{2}}{s}\right)\left(1+2 \alpha\left(M^{\beta}\right)+\frac{\alpha^{2}(1+2 \beta)}{(\beta+1)^{2}} M^{2 \beta}\right) \\
\left.+\frac{1}{2} s I_{e} D M^{2}\left[1-(1-\lambda)\left(\frac{p}{s}\right)\left(1+\frac{\alpha}{\beta+1} M^{\beta}\right)\right]\left[1+(1-\lambda)\left(\frac{p}{s}\right)\left(1+\frac{\alpha(1+2 \beta)}{\beta+1} M^{\beta}\right)\right]-A\right\}
\end{aligned}
$$

and let $\left.\Delta_{6} \equiv \frac{\mathrm{d} T R C_{4}(T)}{\mathrm{d} T}\right|_{T=T_{W}}$, then

$$
\begin{aligned}
\Delta_{6} \equiv & \left\{D h\left[\frac{1}{2} T_{W}^{2}+\frac{\alpha \beta}{\beta+2} T_{W}^{\beta+2}\right]+D p \frac{\alpha \beta}{\beta+1} T_{W}^{\beta+1}+\frac{1}{2} I_{k} D T_{W}^{2}(1-\lambda)^{2}\left(\frac{p^{2}}{s}\right)\left(1+2 \alpha\left(T_{W}^{\beta}\right)+\frac{\alpha^{2}(1+2 \beta)}{(\beta+1)^{2}} T_{W}^{2 \beta}\right)\right. \\
& +\frac{1}{2} p I_{k} D\left[T_{W}^{2}-M^{2}+2 \frac{\alpha \beta}{\beta+2} T_{W}^{\beta+2}+2 \frac{\alpha \beta}{(\beta+1)(\beta+2)} M^{\beta+2}-2 \frac{\alpha \beta}{\beta+1} M T_{W}^{\beta+1}\right] \\
& \left.+\frac{1}{2} S I_{e} D\left[M-(1-\lambda)\left(\frac{p}{s}\right)\left(T_{W}+\frac{\alpha}{\beta+1} T_{W}^{\beta+1}\right)\right]\left[M+(1-\lambda)\left(\frac{p}{s}\right)\left(T_{W}+\frac{\alpha(1+2 \beta)}{\beta+1} T_{W}^{\beta+1}\right)\right]-A\right\}
\end{aligned}
$$

Then we have the following lemma:

\section{Lemma 5:}

a) If $\Delta_{5} \leq 0 \leq \Delta_{6}$, then the annual total relevant cost $T R C_{4}(T)$ has the unique minimum value at the point $T=T_{4}$, where $T_{4} \in\left[M, T_{w}\right)$ and (24) is satisfied by $T_{4}$.

b) If $\Delta_{5}>0$, the annual total relevant cost $T R C_{4}(T)$ has a minimum value at the lower boundary point $T=M$.

c) If $\Delta_{6}<0$, then $T \in\left[M, T_{w}\right)$ which minimizes $T R C_{4}(T)$ does not exist.

Proof: The proof of (a) and (b) is similar to that in Lemma 1 and that of (c) is similar to that in Lemma $3 \mathbf{b}$.

Again in $(0, M]$, the total relevant cost $T R C_{3}(T)$ in (11) is minimum when $\frac{\mathrm{d} T R C_{3}(T)}{\mathrm{d} T}=0$, which is same as in (21), since at $T=M$, then $\Delta_{3}=\Delta_{5}$, now we have the following lemma:

Lemma 6: 
a) If $\Delta_{5} \geq 0$, then the annual total relevant cost $T R C_{3}(T)$ has the unique minimum value at the point $T=T_{3}$, where $T_{3} \in(0, M]$ and satisfies (21).

b) If $\Delta_{5}<0$, the annual total relevant $\operatorname{cost} T R C_{3}(T)$ has a minimum value at the boundary point $T=M$.

Proof: The proof is similar to that in Lemma 1 so we omit it.

From (23) and (26), we get $\Delta_{6} \geq \Delta_{4}$ for $0 \leq \lambda \leq 1$. Again since $M<T_{w}$, we get $\Delta_{6} \geq \Delta_{5}$. Now combining Lemmas 4-6 and the fact that $T R C_{1}(M)=T R C_{2}(M)$, we can obtain a theoretical result to determine the optimal cycle time $T^{*}$ for Case 2 .

Theorem 2:

For $M<T_{w} \leq T_{0}$, the optimal replenishment cycle time $T^{*}$, that minimizes the annual total relevant cost is given as follows:

\begin{tabular}{|c|c|c|c|}
\hline Situation & Condition & $\operatorname{TRC}\left(T^{*}\right)$ & $T^{*}$ \\
\hline$\Delta_{6}<0$ & $\Delta_{4}<0, \Delta_{5}<0$ & $\operatorname{Min}\left\{T R C_{1}\left(T_{1}\right), T R C_{3}(M)\right\}$ & $T_{1}$ or $M$ \\
\hline \multirow{4}{*}{$\Delta_{6} \geq 0$} & $\Delta_{4}<0, \Delta_{5}<0$ & $\operatorname{Min}\left\{T R C_{1}\left(T_{1}\right), T R C_{4}\left(T_{4}\right)\right\}$ & $T_{1}$ or $T_{4}$ \\
\hline & $\Delta_{4}<0, \Delta_{5} \geq 0$ & $\operatorname{Min}\left\{T R C_{1}\left(T_{1}\right), T R C_{3}\left(T_{3}\right)\right\}$ & $T_{1}$ or $T_{3}$ \\
\hline & $\Delta_{4} \geq 0, \Delta_{5}<0$ & $\operatorname{Min}\left\{T R C_{1}\left(T_{w}\right), T R C_{4}\left(T_{4}\right)\right\}$ & $T_{w}$ or $T_{4}$ \\
\hline & $\Delta_{4} \geq 0, \Delta_{5} \geq 0$ & $\operatorname{Min}\left\{T R C_{1}\left(T_{w}\right), T R C_{3}\left(T_{3}\right)\right\}$ & $T_{w}$ or $T_{3}$ \\
\hline
\end{tabular}

\subsection{Case $3 M<T_{0}<T_{w}$}

For $\left[T_{w}, \infty\right)$, the annual total relevant cost is similar as in (9) i.e. $T R C_{1}(T)$. From Lemma 4 of the Case 2, if $\Delta_{4} \leq$ $0, T R C_{1}(T)$ has the unique minimum value at $T=T_{1}$, where $T_{1} \in\left[T_{w}, \infty\right)$ and satisfies (17) and if $\Delta_{4}>0$, then $T R C_{1}(T)$ has minimum value at the boundary point $T=T_{w}$.

Again in $\left[T_{0}, T_{w}\right)$, the annual total relevant cost $T R C_{5}(T)$ in (16) is minimum when $\frac{\mathrm{d} T R C_{5}(T)}{\mathrm{d} T}=0$.

$$
\begin{aligned}
\Rightarrow & \left\{D h\left[\frac{1}{2} T^{2}+\frac{\alpha \beta}{\beta+2} T^{\beta+2}\right]+D p \frac{\alpha \beta}{\beta+1} T^{\beta+1}+\frac{1}{2} I_{k} D T^{2}\left(1-2 \lambda+\lambda^{2}\right)\left(\frac{p^{2}}{s}\right)\left(1+2 \alpha\left(T^{\beta}\right)+\frac{\alpha^{2}(1+2 \beta)}{(\beta+1)^{2}} T^{2 \beta}\right)\right. \\
& \left.+\lambda p I_{k} D\left[(1-\lambda)\left(\frac{p}{s}\right)\left(T^{2}+2 \alpha\left(T^{\beta+2}\right)+\frac{\alpha^{2}(1+2 \beta)}{(\beta+1)^{2}} T^{2(\beta+1)}\right)-\frac{\alpha \beta}{\beta+1} M T^{\beta+1}\right]-A\right\}=0
\end{aligned}
$$

To prove that there exist a value of $T$ in $\left[T_{0}, T_{w}\right)$ at which minimizes $T R C_{5}(T)$, we let $\left.\Delta_{7} \equiv \frac{\mathrm{d} T R C_{5}(T)}{\mathrm{d} T}\right|_{T=T_{0}}$

$$
\begin{aligned}
\Delta_{7}= & \left\{D h\left[\frac{1}{2} T_{0}^{2}+\frac{\alpha \beta}{\beta+2} T_{0}^{\beta+2}\right]+D p \frac{\alpha \beta}{\beta+1} T_{0}^{\beta+1}\right. \\
& +\frac{1}{2} I_{k} D\left(1-2 \lambda+\lambda^{2}\right)\left(\frac{p^{2}}{s}\right)\left(T_{0}^{2}+2 \alpha\left(T_{0}^{\beta+2}\right)+\frac{\alpha^{2}(1+2 \beta)}{(\beta+1)^{2}} T_{0}^{2(\beta+1)}\right) \\
& \left.+\lambda p I_{k} D\left[(1-\lambda)\left(\frac{p}{s}\right)\left(T_{0}^{2}+2 \alpha\left(T_{0}^{\beta+2}\right)+\frac{\alpha^{2}(1+2 \beta)}{(\beta+1)^{2}} T_{0}^{2(\beta+1)}\right)-\frac{\alpha \beta}{\beta+1} M T_{0}^{(\beta+1)}\right]-A\right\}
\end{aligned}
$$

and

$$
\left.\Delta_{8} \equiv \frac{\mathrm{d} T R C_{5}(T)}{\mathrm{d} T}\right|_{T=T_{W}}
$$




$$
\begin{aligned}
\Delta_{8}= & \left\{D h\left[\frac{1}{2} T_{W}^{2}+\frac{\alpha \beta}{\beta+2} T_{W}^{\beta+2}\right]+D p \frac{\alpha \beta}{\beta+1} T_{W}^{\beta+1}\right. \\
& +\frac{1}{2} I_{k} D\left(1-2 \lambda+\lambda^{2}\right)\left(\frac{p^{2}}{s}\right)\left(T_{W}^{2}+2 \alpha\left(T_{W}^{\beta+2}\right)+\frac{\alpha^{2}(1+2 \beta)}{(\beta+1)^{2}} T_{W}^{2(\beta+1)}\right) \\
& \left.+\lambda p I_{k} D\left[(1-\lambda)\left(\frac{p}{s}\right)\left(T_{W}^{2}+2 \alpha\left(T_{W}^{\beta+2}\right)+\frac{\alpha^{2}(1+2 \beta)}{(\beta+1)^{2}} T_{W}^{2(\beta+1)}\right)-\frac{\alpha \beta}{\beta+1} M T_{W}^{(\beta+1)}\right]-A\right\}
\end{aligned}
$$

Consequently, we have the following lemma:

\section{Lemma 7:}

a) If $\Delta_{7} \leq 0 \leq \Delta_{8}$, then the annual total relevant cost $T R C_{5}(T)$ has the unique minimum value at the point $T=T_{5}$, where $T_{5} \in\left[T_{0}, T_{w}\right.$ ) and (27) is satisfied by $T_{5}$.

b) If $\Delta_{7}>0$, the annual total relevant cost $T R C_{5}(T)$ has a minimum value at the lower boundary point $T=T_{0}$.

c) If $\Delta_{8}<0$, then $T \in\left[T_{0}, T_{w}\right.$ ) which minimizes $T R C_{5}(T)$ does not exist.

Proof: The proof of a) and b) is similar to that in Lemma 1 and that of (c) is similar to that in Lemma $3 \mathbf{b}$.

Again in $\left[M, T_{0}\right]$, the annual relevant cost is similar to $T R C_{4}(T)$ in (13). $T R C_{4}(T)$ is minimum when $\frac{{\mathrm{d} T R C_{4}}_{4}(T)}{\mathrm{d} T}=0$, which is same as in (24).

To prove that there exists a unique value of $T$ in $\left[M, T_{0}\right]$ at which minimizes $\operatorname{TRC}_{4}(T)$, we let

$$
\begin{aligned}
\Delta_{9}=\left.\frac{\mathrm{d} T R C_{4}(T)}{\mathrm{d} T}\right|_{T=T_{0}} & \Delta_{9}=\left\{D h\left[\frac{1}{2} T_{0}^{2}+\frac{\alpha \beta}{\beta+2} T_{0}^{\beta+2}\right]+D p \frac{\alpha \beta}{\beta+1} T_{0}^{\beta+1}+\frac{1}{2} I_{k} D T_{0}^{2}(1-\lambda)^{2}\left(\frac{p^{2}}{s}\right)\left(1+2 \alpha\left(T_{0}^{\beta}\right)+\frac{\alpha^{2}(1+2 \beta)}{(\beta+1)^{2}} T_{0}^{2 \beta}\right)\right. \\
& +\frac{1}{2} I_{k} D\left[T_{0}^{2}-M^{2}+\frac{2 \alpha(\beta)}{(\beta+2)} T_{0}^{(\beta+2)}+\frac{2 \alpha(\beta)}{(\beta+1)(\beta+2)} M^{(\beta+2)}-\frac{2 \alpha(\beta)}{(\beta+1)} M T_{0}^{\beta+1}\right] \\
& \left.+\frac{1}{2} s D I_{e}\left[M-(1-\lambda)\left(\frac{p}{s}\right)\left(T_{0} \frac{\alpha}{(\beta+1)} T_{0}^{\beta+1}\right)\right] \times\left[M+(1-\lambda)\left(\frac{p}{s}\right)\left(T_{0} \frac{\alpha(1+2 \beta)}{(\beta+1)} T_{0}^{\beta+1}\right)\right]-A\right\}
\end{aligned}
$$

Then we have the following lemma:

\section{Lemma 8:}

a) If $\Delta_{5} \leq 0 \leq \Delta_{9}$, then the annual total relevant cost $T R C_{4}(T)$ has the unique minimum value at the point $T=T_{4}$, where $T_{4} \in\left[M, T_{0}\right]$ and (24) is satisfied by $T_{4}$.

b) If $\Delta_{5}>0$, the annual total relevant cost $T R C_{4}(T)$ has a minimum value at the lower boundary point $T=M$.

c) If $\Delta_{9}<0$, the annual total relevant cost $T R C_{4}(T)$ has a minimum value at the upper boundary point $T=T_{0}$.

Proof: The proof is similar to that in Lemma 1 so we omit it.

Again in $(0, M]$, the annual total relevant cost is similar to $T R C_{3}(T)$ in (15). We know that at $T=M, \Delta_{3}=\Delta_{5}$, so from Lemma 6, if $\Delta_{5} \geq 0, T R C_{3}(T)$ has unique minimum value at $T=T_{3}$, where $T_{3} \in(0, M)$ and satisfies (21). On the other hand if $\Delta_{5}<0$, then $T R C_{3}(T)$ has a minimum value at boundary point $T=M$.

Since $M<T_{0}<T_{w}$, from (28) and (30) we can get $\Delta_{7} \leq \Delta_{9}$. Again we know that $\Delta_{5} \leq \Delta_{9}$ and $\Delta_{5} \leq \Delta_{7} \leq \Delta_{8}$ for 0 $\leq \lambda \leq 1$. Consequently, combining Lemmas 4, 6, 7 and 8, and the fact that $T R C_{3}(M)=T R C_{4}(M)$, we can obtain the theoretical result to get the optimal cycle time $T^{*}$ for Case 3 as:

\section{Theorem 3:}

For $M<T_{0}<T_{w}$, the optimal replenishment cycle time $T^{*}$, that minimizes the annual total relevant cost is given as follows: 


\begin{tabular}{|c|c|c|c|c|}
\hline Situation & Condition & Sub-condition & $\operatorname{TRC}\left(T^{*}\right)$ & $T^{*}$ \\
\hline \multirow{6}{*}{$\Delta_{4}<0$} & \multirow{6}{*}{$\begin{array}{l}\Delta_{8}<0, \Delta_{9}<0 \\
\Delta_{8}<0, \Delta_{9} \geq 0 \\
\Delta_{8} \geq 0, \Delta_{9}<0 \\
\Delta_{8} \geq 0, \Delta_{9} \geq 0\end{array}$} & & $\operatorname{Min}\left\{T R C_{1}\left(T_{1}\right), T R C_{4}\left(T_{0}\right)\right\}$ & $T_{1}$ or $T_{0}$ \\
\hline & & & $\operatorname{Min}\left\{T R C_{1}\left(T_{1}\right), T R C_{4}\left(T_{4}\right)\right\}$ & $T_{1}$ or $T_{4}$ \\
\hline & & & $\operatorname{Min}\left\{T R C_{1}\left(T_{1}\right), T R C_{5}\left(T_{5}\right), T R C_{4}\left(T_{0}\right)\right\}$ & $T_{1}$ or $T_{5}$ or $T_{0}$ \\
\hline & & & $\operatorname{Min}\left\{T R C_{1}\left(T_{1}\right), T R C_{5}\left(T_{5}\right), T R C_{4}\left(T_{4}\right)\right\}$ & $T_{1}$ or $T_{5}$ or $T_{4}$ \\
\hline & & & $\operatorname{Min}\left\{T R C_{1}\left(T_{1}\right), T R C_{5}\left(T_{0}\right), T R C_{4}\left(T_{4}\right)\right\}$ & $T_{1}$ or $T_{0}$ or $T_{4}$ \\
\hline & & & $\operatorname{Min}\left\{T R C_{1}\left(T_{1}\right), T R C_{5}\left(T_{0}\right), T R C_{3}\left(T_{3}\right)\right\}$ & $T_{1}$ or $T_{0}$ or $T_{3}$ \\
\hline \multirow{6}{*}{$\Delta_{4} \geq 0$} & \multirow{6}{*}{$\begin{array}{l}\Delta_{8}<0, \Delta_{9}<0 \\
\Delta_{8}<0, \Delta_{9} \geq 0 \\
\Delta_{8} \geq 0, \Delta_{9}<0 \\
\Delta_{8} \geq 0, \Delta_{9} \geq 0\end{array}$} & & $\operatorname{Min}\left\{T R C_{1}\left(T_{w}\right), T R C_{4}\left(T_{0}\right)\right\}$ & $T_{w}$ or $T_{0}$ \\
\hline & & & $\operatorname{Min}\left\{T R C_{1}\left(T_{w}\right), T R C_{4}\left(T_{4}\right)\right\}$ & $T_{w}$ or $T_{4}$ \\
\hline & & & $\operatorname{Min}\left\{T R C_{1}\left(T_{w}\right), T R C_{5}\left(T_{5}\right), T R C_{4}\left(T_{0}\right)\right\}$ & $T_{w}$ or $T_{5}$ or $T_{0}$ \\
\hline & & & $\operatorname{Min}\left\{T R C_{1}\left(T_{w}\right), T R C_{5}\left(T_{5}\right), T R C_{4}\left(T_{4}\right)\right\}$ & $T_{w}$ or $T_{5}$ or $T_{4}$ \\
\hline & & & $\operatorname{Min}\left\{T R C_{1}\left(T_{w}\right), T R C_{5}\left(T_{0}\right), T R C_{4}\left(T_{4}\right)\right\}$ & $T_{w}$ or $T_{0}$ or $T_{4}$ \\
\hline & & & $\operatorname{Min}\left\{T R C_{1}\left(T_{w}\right), T R C_{5}\left(T_{0}\right), T R C_{3}\left(T_{3}\right)\right\}$ & $T_{w}$ or $T_{0}$ or $T_{3}$ \\
\hline
\end{tabular}

\section{Solution Procedures}

Here we develop the following algorithm to solve this complex inventory problem by using the characteristics of Theorems 1-3 above.

\section{Algorithm:}

Step 1 Compare the values of $T_{0}, M$ and $T_{w}$, if $T_{w} \leq M<T_{0}$, then go to step 2 .

If $M<T_{w} \leq T_{0}$, then go to Step 3. Otherwise, if $M<T_{0}<T_{w}$, then go to Step 4 .

Calculate $\Delta_{1}, \Delta_{2}$ and $\Delta_{3}$ which are shown as in Equation (18), (20) and (22), respectively.

1) If $\Delta_{1}<0$ and $\Delta_{3}<0$, then $T R C\left(T^{*}\right)=T R C_{1}\left(T_{1}\right)$ and $T^{*}=T_{1}$. Go to Step 5 .

Step 2 2) If $\Delta_{1}<0$ and $\Delta_{3} \geq 0$, then $\operatorname{TRC}\left(T^{*}\right)=\min \left\{T R C_{1}\left(T_{1}\right), T R C_{3}\left(T_{3}\right)\right\}$ and $T^{*}=T_{1}$ or $T_{3}$. Go to Step 5 .

3) If $\Delta_{1} \geq 0, \Delta_{2}<0$ and $\Delta_{3} \geq 0$, then $\operatorname{TRC}\left(T^{*}\right)=\min \left\{T R C_{2}\left(T_{2}\right), T R C_{3}\left(T_{3}\right)\right\}$ and $T^{*}=T_{2}$ or $T_{3}$. Go to Step 5 .

4) If $\Delta_{2} \geq 0$, then $\operatorname{TRC}\left(T^{*}\right)=\min \left\{T R C_{2}\left(T_{w}\right), T R C_{3}\left(T_{3}\right)\right\}$ and $T^{*}=T_{w}$ or $T_{3}$. Go to Step 5 .

5) If $\Delta_{1} \geq 0$ and $\Delta_{3}<0$, then $\operatorname{TRC}\left(T^{*}\right)=T R C_{2}\left(T_{2}\right)$ and $T^{*}=T_{2}$. Go to Step 5 .

Calculate $\Delta_{4}, \Delta_{5}$ and $\Delta_{6}$ which are shown as in Equations (23), (25) and (26), respectively.

1) If $\Delta_{6}<0$, then $T R C\left(T^{*}\right)=\min \left\{T R C_{1}\left(T_{1}\right), T R C_{3}(\mathrm{M})\right\}$ and $T^{*}=T_{1}$ or M. Go to Step 5 .

Step 32 2) If $\Delta_{4}<0, \Delta_{5}<0$ and $\Delta_{6} \geq 0$, then $T R C\left(T^{*}\right)=\min \left\{T R C_{1}\left(T_{1}\right), T R C_{4}\left(T_{4}\right)\right\}$ and $T^{*}=T_{1}$ or $T_{4}$. Go to Step 5.

3) If $\Delta_{4}<0$ and $\Delta_{5} \geq 0$, then $\operatorname{TRC}\left(T^{*}\right)=\min \left\{T R C_{1}\left(T_{1}\right), T R C_{3}\left(T_{3}\right)\right\}$ and $T^{*}=T_{1}$ or $T_{3}$. Go to Step 5 .

4) If $\Delta_{4} \geq 0$ and $\Delta_{5}<0$, then $\operatorname{TRC}\left(T^{*}\right)=\min \left\{T R C_{1}\left(T_{w}\right), T R C_{4}\left(T_{4}\right)\right\}$ and $T^{*}=T_{w}$ or $T_{4}$. Go to Step 5.

5) If $\Delta_{4} \geq 0$ and $\Delta_{5} \geq 0$, then $\operatorname{TRC}\left(T^{*}\right)=\min \left\{T R C_{1}\left(T_{w}\right), T R C_{3}\left(T_{3}\right)\right\}$ and $T^{*}=T_{w}$ or $T_{3}$. Go to Step 5 .

Step 4 Calculate $\Delta_{4}, \Delta_{5}, \Delta_{7}, \Delta_{8}$ and $\Delta_{9}$ which are shown as in Equation (23), (25), (28), (29) and (30) respectively.

If $\Delta_{4}<0$, then go to Step 4.1. Otherwise, go to Step 4.2.

1) If $\Delta_{8}<0$ and $\Delta_{9}<0$, then $\operatorname{TRC}\left(T^{*}\right)=\min \left\{T R C_{1}\left(T_{1}\right), T R C_{4}\left(T_{0}\right)\right\}$ and $T^{*}=T_{1}$ or $T_{0}$. Go to Step 5 .

2) If $\Delta_{8}<0$ and $\Delta_{9} \geq 0$, then $\operatorname{TRC}\left(T^{*}\right)=\min \left\{T R C_{1}\left(T_{1}\right), T R C_{4}\left(T_{4}\right)\right\}$ and $T^{*}=T_{1}$ or $T_{4}$. Go to Step 5.

3) If $\Delta_{8} \geq 0$ and $\Delta_{9}<0$, then $\operatorname{TRC}\left(T^{*}\right)=\min \left\{T R C_{1}\left(T_{1}\right), T R C_{4}\left(T_{0}\right), T R C_{5}\left(T_{5}\right)\right\}$ and $T^{*}=T_{1}$ or $T_{0}$ or $T_{5}$. Go to Step 5 .

4) If $\Delta_{7}<0, \Delta_{8} \geq 0$ and $\Delta_{9} \geq 0$, then $\operatorname{TRC}\left(T^{*}\right)=\min \left\{T R C_{1}\left(T_{1}\right), T R C_{4}\left(T_{4}\right), T R C_{5}\left(T_{5}\right)\right\}$ and $T^{*}=T_{1}$ or $T_{4}$ or $T_{5}$. Go to Step 5.

5) If $\Delta_{5}<0$ and $\Delta_{7} \geq 0$, then $\operatorname{TRC}\left(T^{*}\right)=\min \left\{T R C_{1}\left(T_{1}\right), T R C_{4}\left(T_{4}\right), T R C_{5}\left(T_{0}\right)\right\}$ and $T^{*}=T_{1}$ or $T_{4}$ or $T_{0}$. Go to Step 5 .

6) If $\Delta_{5} \geq 0$, then $\operatorname{TRC}\left(T^{*}\right)=\min \left\{T R C_{1}\left(T_{1}\right), T R C_{3}\left(T_{3}\right), T R C_{5}\left(T_{0}\right)\right\}$ and $T^{*}=T_{1}$ or $T_{3}$ or $T_{0}$. Go to Step 5 .

Step 4.17$)$ If $\Delta_{8}<0$ and $\Delta_{9}<0$, then $T R C\left(T^{*}\right)=\min \left\{T R C_{1}\left(T_{1}\right), T R C_{4}\left(T_{0}\right)\right\}$ and $T^{*}=T_{1}$ or $T_{0}$. Go to Step 5 .

8) If $\Delta_{8}<0$ and $\Delta_{9} \geq 0$, then $\operatorname{TRC}\left(T^{*}\right)=\min \left\{T R C_{1}\left(T_{1}\right), T R C_{4}\left(T_{4}\right)\right\}$ and $T^{*}=T_{1}$ or $T_{4}$. Go to Step 5.

9) If $\Delta_{8} \geq 0$ and $\Delta_{9}<0$, then $\operatorname{TRC}\left(T^{*}\right)=\min \left\{T R C_{1}\left(T_{1}\right), T R C_{4}\left(T_{0}\right), T R C_{5}\left(T_{5}\right)\right\}$ and $T^{*}=T_{1}$ or $T_{0}$ or $T_{5}$. Go to Step 5 .

10) If $\Delta_{7}<0, \Delta_{8} \geq 0$ and $\Delta_{9} \geq 0$, then $\operatorname{TRC}\left(T^{*}\right)=\min \left\{T R C_{1}\left(T_{1}\right), T R C_{4}\left(T_{4}\right), T R C_{5}\left(T_{5}\right)\right\}$ and $T^{*}=T_{1}$ or $T_{4}$ or $T_{5}$. Go to Step 5.

11) If $\Delta_{5}<0$ and $\Delta_{7} \geq 0$, then $\operatorname{TRC}\left(T^{*}\right)=\min \left\{T R C_{1}\left(T_{1}\right), T R C_{4}\left(T_{4}\right), T R C_{5}\left(T_{0}\right)\right\}$ and $T^{*}=T_{1}$ or $T_{4}$ or $T_{0}$. Go to Step 5 .

12) If $\Delta_{5} \geq 0$, then $\operatorname{TRC}\left(T^{*}\right)=\min \left\{T R C_{1}\left(T_{1}\right), T R C_{3}\left(T_{3}\right), T R C_{5}\left(T_{0}\right)\right\}$ and $T^{*}=T_{1}$ or $T_{3}$ or $T_{0}$. Go to Step 5 .

1) If $\Delta_{8}<0$ and $\Delta_{9}<0$, then $\operatorname{TRC}\left(T^{*}\right)=\min \left\{T R C_{1}\left(T_{w}\right), T R C_{4}\left(T_{0}\right)\right\}$ and $T^{*}=T_{w}$ or $T_{0}$. Go to Step 5 .

2) If $\Delta_{8}<0$ and $\Delta_{9} \geq 0$, then $\operatorname{TRC}\left(T^{*}\right)=\min \left\{T R C_{1}\left(T_{w}\right), T R C_{4}\left(T_{4}\right)\right\}$ and $T^{*}=T_{w}$ or $T_{4}$. Go to Step 5.

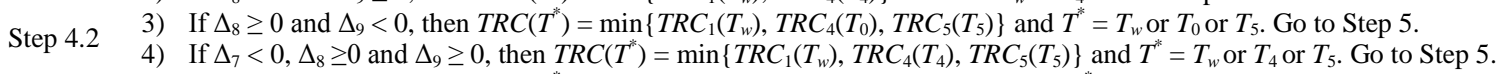

5) If $\Delta_{5}<0$ and $\Delta_{7} \geq 0$, then $\operatorname{TRC}\left(T^{*}\right)=\min \left\{T R C_{1}\left(T_{w}\right), T R C_{4}\left(T_{4}\right), T R C_{5}\left(T_{0}\right)\right\}$ and $T^{*}=T_{w}$ or $T_{4}$ or $T_{0}$. Go to Step 5 .

6) If $\Delta_{5} \geq 0$, then $\operatorname{TRC}\left(T^{*}\right)=\min \left\{T R C_{1}\left(T_{w}\right), T R C_{3}\left(T_{3}\right), T R C_{5}\left(T_{0}\right)\right\}$ and $T^{*}=T_{w}$ or $T_{3}$ or $T_{0}$. Go to Step 5. 


\section{Numerical Example}

In this section, the present study provides the following numerical example as shown in Huang [5] to illustrate all the theoretical results. The values of the parameters are taken randomly.

We assume that selling price per unit $s=\$ 50$, ordering cost $A=\$ 50 /$ order, demand $D=1000$ units/year, purchasing cost $p=\$ 20$, holding cost $h=\$ 5 /$ unit/year, period of permissible delay $M=0.12$ year, interest earned $I_{e}$ $=\$ 0.07 / \$ /$ year, interest charged $I_{k}=\$ 0.1 / \$ /$ year, scale parameter $\alpha=0.02$, shape parameter $\beta=1.5$.

We obtain the optimal cycle time and optimal order quantity for different parameters of the fraction of the delay payment $\lambda=\{0.2,0.5,0.8\}$ and the prefix quantity $\mathrm{W}=\{50,150,250\}$ as shown in Table 1 .

\section{Sensitivity Analysis}

The purpose of the sensitivity analysis is to identify parameters to the changes of which the solution of the model is sensitive. The following inferences can be made based on above solution table.

Table 1. Optimal solutions of deterministic model under different parametric values.

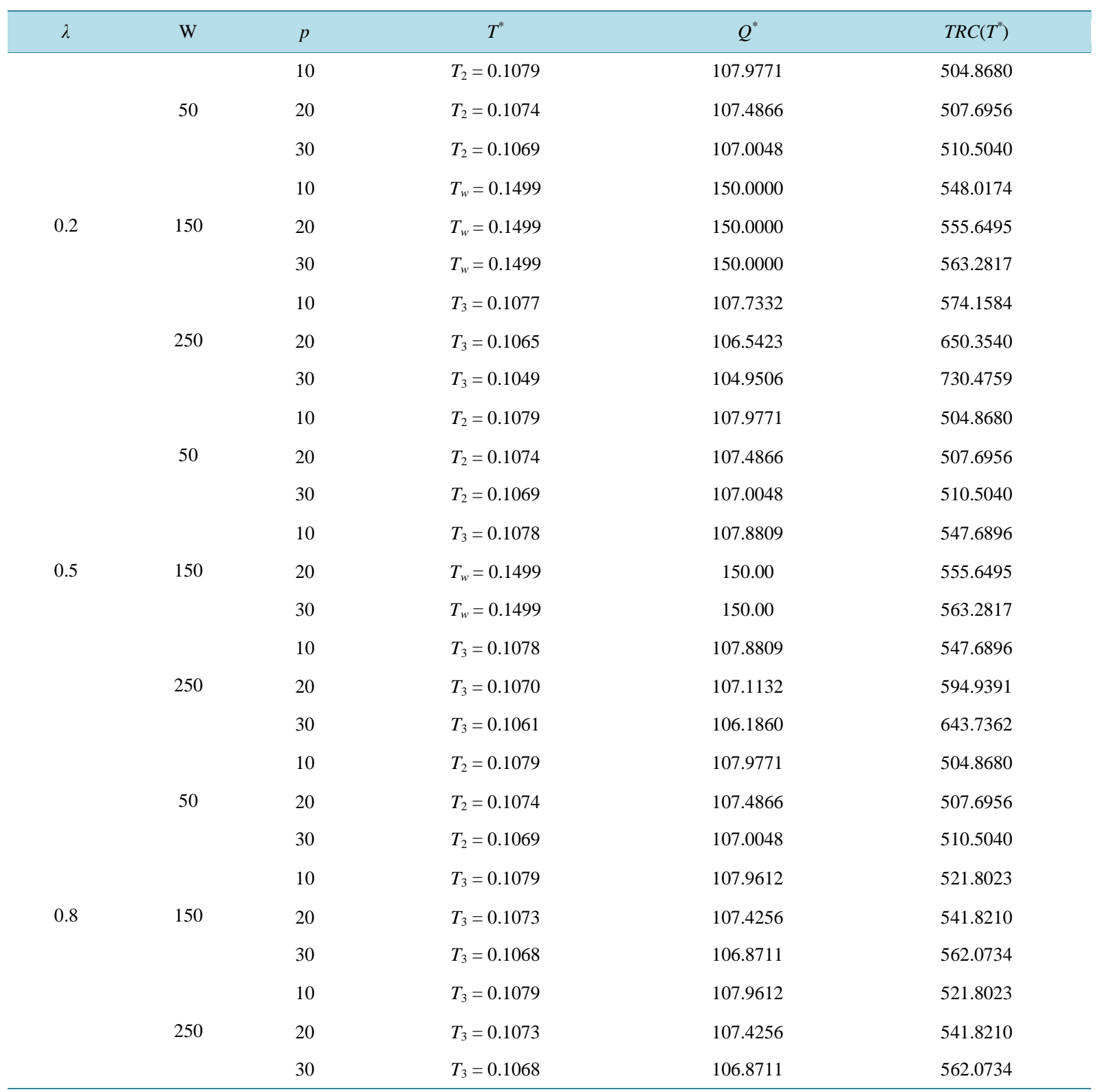


1) For fixed $\mathrm{W}$ and $p$, increasing the value of $\lambda$ will result in a significant increase in the value of the optimal order quantity and a significant decrease in the value of the annual total relevant costs as the retailer's order quantity is smaller and only the partially delayed payment is permitted.

For example when $\mathbf{W}=\mathbf{2 5 0}, \boldsymbol{p}=\mathbf{3 0}$ and $\lambda$ increases from $\mathbf{0 . 2}$ to $\mathbf{0 . 5}$, the optimal order quantity will increase 1.17\%((106.1860 - 104.9506)/104.9506) and the annual total relevant costs will decrease $11.87 \%((730.4759-$ 643.7362)/730.4759). However, if the fully delayed payment is permitted, the optimal order quantity and the annual total relevant cost are independent of the value of $\lambda$. It implies that the retailer will order a larger quantity since the retailer can enjoy greater benefits when the fraction of the delay payments permitted is increasing. So the supplier can use the policy of increasing $\lambda$ to stimulate the demands from the retailer. Consequently, the supplier's marketing policy under partially permissible delay in payments will be more attractive than fully permissible delay in payments.

2) For fixed $\lambda$ and $p$, increasing the value of $\mathrm{W}$ will result in a significant decrease in the value of the optimal order quantity and a significant increase in the value of the annual total relevant costs.

For example, when $\lambda=\mathbf{0 . 2}, \boldsymbol{p}=\mathbf{3 0}$ and $\mathrm{W}$ increases from $\mathbf{1 5 0}$ to $\mathbf{2 5 0}$, the optimal order quantity will decrease

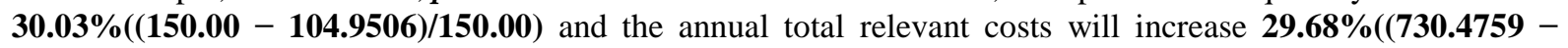
563.2817)/563.2817). It implies that the retailer will not order a quantity as large as the minimum order quantity as required to obtain fully permissible delay in payments. Hence, the effect of stimulating the demands from the retailer turns negative when the supplier adopts a policy to increase the value of $\mathrm{W}$.

3) Last, for fixed $\lambda$ and $\mathrm{W}$, increasing the value of $p$ will result in a significant decrease in the value of the optimal order quantity and a significant increase in the value of the annual total relevant cost. However, for the case with $\lambda=0.2$ and $\mathrm{W}=150$ in the numerical example, the optimal replenishment cycle and optimal order quantity are fixed and are not affected by the increase of the unit purchase price. The reason is that in this situation, the retailer trades off the benefits of full delay in payment against the partial delay in payment and enjoys the full delay in payment.

\section{ANOVA Analysis}

If the values of $\lambda$ and $\mathrm{W}$ are taken randomly (Table 2), the Two-way ANOVA analysis on Total Relevant Cost (TRC) shown in Table 3:

\subsection{Does W Value Affect the Result?}

Since from Table 3, the calculated values are $\mathrm{F}_{\text {cal }}=8.17, \mathrm{df}_{\mathrm{n}}=2, \mathrm{df}_{\mathrm{d}}=4, \alpha=0.05, \mathrm{~F}_{\text {table }}=6.9443$ and $\mathrm{F}_{\text {cal }}>\mathrm{F}_{\text {table, }}$, we conclude that effect of prefixed quantity (W value) on the result (Total relevant cost) is considered extremely significant.

Table 2. Cost table: (when $p=10)$.

\begin{tabular}{cccc} 
& $\mathrm{W}=50$ & $\mathrm{~W}-=150$ & $\mathrm{~W}=250$ \\
\hline$\lambda=0.2$ & 504.8680 & 548.0174 & 574.1584 \\
$\lambda=0.5$ & 504.8680 & 547.6896 & 547.6896 \\
$\lambda=0.8$ & 504.8680 & 521.8023 & 521.8023 \\
\hline
\end{tabular}

Table 3. Two way ANOVA table.

\begin{tabular}{ccccc}
\hline Source of Variation & df & Sum-of-squares & Mean square & Calculated F $F_{\text {cal }}$ value \\
\hline W value & 2 & 3103 & 1551 & 8.170 \\
Fraction of permissible delay & 2 & 1064 & 531.8 & 2.800 \\
Residual (error) & 4 & 759.6 & 189.9 \\
Total & 8 & 4926 & \\
\hline
\end{tabular}




\subsection{Does Fraction of Permissible Delay Affect the Result?}

From the calculated values of Table 4, $\mathrm{F}_{\text {cal }}=2.80, \mathrm{df}_{\mathrm{n}}=2, \mathrm{df}_{\mathrm{d}}=4, \alpha=0.05, \mathrm{~F}_{\text {table }}=6.9443$ and since $\mathrm{F}_{\text {cal }}<\mathrm{F}_{\text {table, }}$, we can conclude that effect of fraction of permissible delay ( $\lambda$ value) on the result (Total relevant cost) is considered not quite significant.

From the above analysis we can conclude that the fraction of permissible delay has no effect overall i.e., the effect is considered not significant.

\subsection{Does W Value Affect the Result?}

Since from Table 5 the calculated values of $\mathrm{F}_{\text {cal }}=6.611, \mathrm{df}_{\mathrm{n}}=2, \mathrm{df}_{\mathrm{d}}=4, \alpha=0.05, \mathrm{~F}_{\text {table }}=6.9443$ and $\mathrm{F}_{\text {cal }}<\mathrm{F}_{\text {table, }}$, we conclude that effect of prefixed quantity ( $\mathrm{W}$ value) on the result (Total relevant cost) is considered not quite significant.

\subsection{Does Fraction of Permissible Delay Affect the Result?}

From the calculated values of Table $6, \mathrm{~F}_{\text {cal }}=1.424, \mathrm{df}_{\mathrm{n}}=2, \mathrm{df}_{\mathrm{d}}=4, \alpha=0.05, \mathrm{~F}_{\text {table }}=6.9443$ and $\mathrm{F}_{\text {cal }}<\mathrm{F}_{\text {table, }}$, we can conclude that effect of fraction of permissible delay ( $\lambda$ value) on the result (Total relevant cost) is considered not quite significant.

From the above analysis we can conclude that after increasing the price rate the effect of $\mathrm{W}$ value and the fraction of permissible delay on the result is considered not significant overall i.e., the effect is considered not significant.

\subsection{Does W Value Affect the Result?}

From the values of Table 7, $\mathrm{F}_{\text {cal }}=5.913, \mathrm{df}_{\mathrm{n}}=2, \mathrm{df}_{\mathrm{d}}=4, \alpha=0.05, \mathrm{~F}_{\text {table }}=6.9443$ and $\mathrm{F}_{\text {cal }}<\mathrm{F}_{\text {table, }}$, we can conclude that effect of prefixed quantity (W value) on the result (Total relevant cost) is considered not quite significant.

Table 4. Cost table: (when $p=20$ ).

\begin{tabular}{cccc}
\hline & $\mathrm{W}=50$ & $\mathrm{~W}-=150$ & $\mathrm{~W}=250$ \\
$\lambda=0.2$ & 507.6956 & 555.6495 & 650.3540 \\
$\lambda=0.5$ & 507.6956 & 555.6495 & 594.9391 \\
$\lambda=0.8$ & 507.6956 & 541.8210 & 541.8210 \\
\hline
\end{tabular}

Table 5. Two way ANOVA table.

\begin{tabular}{ccccc}
\hline Source of Variation & df & Sum-of-squares & Mean square & Calculated $F_{\text {cal }}$ value \\
W value & 2 & 11620 & 5810 & 6.611 \\
Fraction of permissible delay & 2 & 2503 & 1251 & 6.9443 \\
Residual (error) & 4 & 3515 & 878.8 \\
Total & 8 & 17640 & \\
\hline
\end{tabular}

Table 6. Cost table: (when $p=30$ ).

\begin{tabular}{lrrr}
\hline & $\mathrm{W}=50$ & $\mathrm{~W}-=150$ & $\mathrm{~W}=250$ \\
$\lambda=0.2$ & 510.5040 & 563.2817 & 730.4759 \\
$\lambda=0.5$ & 510.5040 & 563.2817 & 643.7362 \\
$\lambda=0.8$ & 510.5040 & 562.0734 & 562.0734
\end{tabular}


Table 7. Two way ANOVA table.

\begin{tabular}{cccccc}
\hline Source of Variation & $\mathrm{df}$ & Sum-of-squares & Mean square & Calculated $\mathrm{F}_{\text {cal }}$ value & Tabular $\mathrm{F}_{\text {table }}$ value \\
\hline W value & 2 & 27760 & 13880 & 5.913 & 6.9443 \\
Fraction of permissible delay & 2 & 4795 & 2398 & 1.021 & 6.9443 \\
Residual (error) & 4 & 9390 & 2347 & & \\
Total & 8 & 41950 & & \\
\hline
\end{tabular}

\subsection{Does Fraction of Permissible Delay Affect the Result?}

Since $\mathrm{F}_{\text {cal }}=1.021, \mathrm{df}_{\mathrm{n}}=2, \mathrm{df}_{\mathrm{d}}=4, \alpha=0.05$ and $\mathrm{F}_{\text {table }}=6.9443$ and $\mathrm{F}_{\text {cal }}<\mathrm{F}_{\text {table }}$, we also conclude that effect of fraction of permissible delay ( $\lambda$ value) on the result (Total relevant cost) is considered not quite significant.

From the above analysis we can conclude that after increasing the price rate the effect of $\mathrm{W}$ value and the fraction of permissible delay on the result is considered not significant overall i.e., the effect is considered not significant.

Data 1

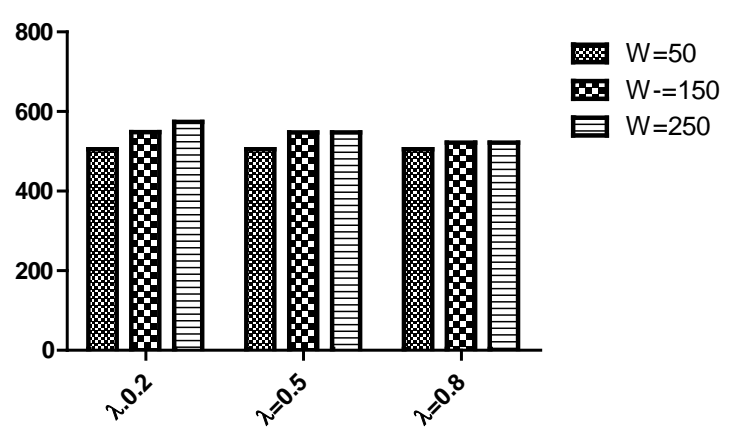

Data 2

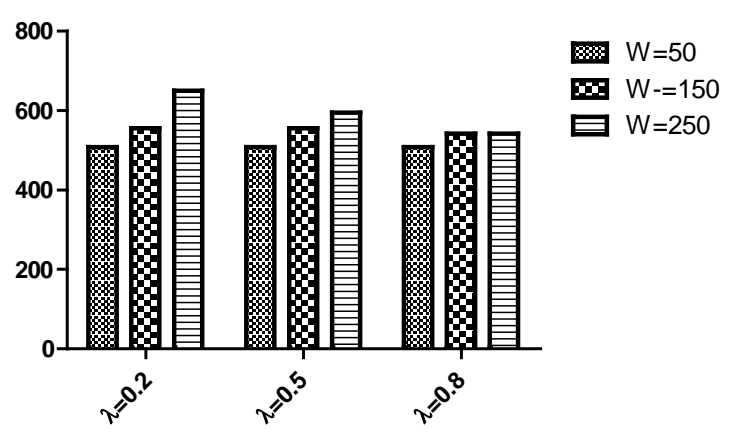

Data 3

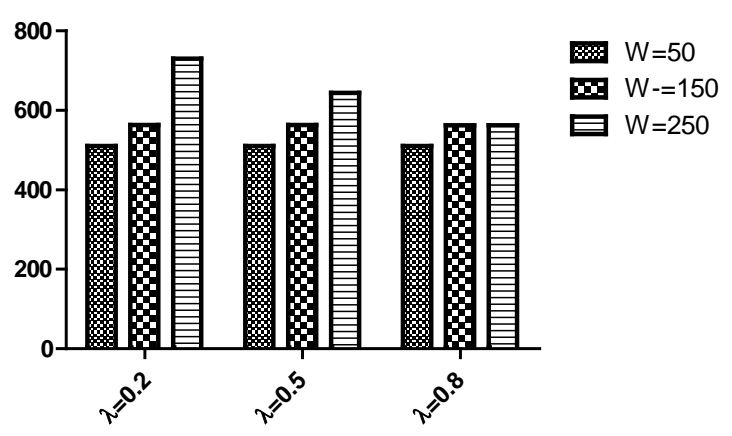




\section{Conclusion}

In this paper, we develop a deterministic inventory model under the conditions of permissible delay in payments by considering the following situations simultaneously: 1) the retailer's selling price per unit is higher than the purchase price; 2) the interest charged by a bank is not necessarily higher than the retailer's investment return rate; 3) many selling items deteriorate continuously such as fresh fruits and vegetables and 4) the supplier may offer a partial permissible delay in payments even if the order quantity is less than W. Considering all these facts, this inventory model has been developed to make more realistic and flexible marketing policy to the retailer.

\section{References}

[1] Goyal, S.K. (1985) Economic Order Quantity under Conditions of Permissible Delay in Payments. Journal of the Operational Research Society (JORS), 36, 335-338. http://dx.doi.org/10.1057/jors.1985.56

[2] Aggarwal, S.P. and Jaggy, C.K. (1995) Ordering Policies of Deterioration Items under Permissible Delay in Payments. Journal of the Operational Research Society (JORS), 46, 658-662. http://dx.doi.org/10.1057/jors.1995.90

[3] Chang, C.T., Ouyang, L.Y. and Teng, J.T. (2003) An EOQ Model for Deteriorating Items under Supplier Credits Linked to Ordering Quantity. Applied Mathematical Modelling (AMM), 27, 983-996. http://dx.doi.org/10.1016/S0307-904X(03)00131-8

[4] Chang, K.J. and Liao, J.J. (2004) Lot-Sizing Decisions under Trade Credit Depending on the Ordering Quantity. Computers \& Operations Research (COR), 31, 909-928. http://dx.doi.org/10.1016/S0305-0548(03)00043-1

[5] Huang, Y.F. (2007) Economic Order Quantity under Conditionally Permissible Delay in Payments. European Journal of Operational Research (EJOR), 176, 911-924. http://dx.doi.org/10.1016/j.ejor.2005.08.017

[6] Ouyang, L.Y., Teng, J.T., Goyal, S.K. and Yang, C.T. (2009) An Economic Order Quantity Model for Deteriorating Items with Partially Permissible Delay in Payments Linked to Order Quantity. European Journal of Operational Research, 194, 418-431. http://dx.doi.org/10.1016/j.ejor.2007.12.018

[7] Das, D., Roy, A. and Kar, S. (2010) Improving Production Policy for a Deteriorating Item under Permissible Delay in Payments with Stock-Dependent Demand Rate. Computers and Mathematics with Applications, 60, 1973-1985. http://dx.doi.org/10.1016/j.camwa.2010.07.031

[8] Teng, J.T., Krommyda, I.P., Skouri, K. and Lou, K.R. (2011) A Comprehensive Extension of Optimal Ordering Policy for Stock-Dependent Demand under Progressive Payment Scheme. European Journal of Operational Research, 215, 97-104. http://dx.doi.org/10.1016/j.ejor.2011.05.056

[9] Min, J., Zhou, Y.W., Liu, G.Q. and Wang, S.D. (2012) An EPQ Model for Deteriorating Items with Inventory-LevelDependent Demand and Permissible Delay in Payments. International Journal of Systems Science, 43, 1039-1053. http://dx.doi.org/10.1080/00207721.2012.659685

[10] Ouyang, L.Y. and Chang, C.T. (2013) Optimal Production Lot with Imperfect Production Process under Permissible Delay in Payments and Complete Backlogging. International Journal of Production Economics, 144, 610-617. http://dx.doi.org/10.1016/j.ijpe.2013.04.027 
Scientific Research Publishing (SCIRP) is one of the largest Open Access journal publishers. It is currently publishing more than 200 open access, online, peer-reviewed journals covering a wide range of academic disciplines. SCIRP serves the worldwide academic communities and contributes to the progress and application of science with its publication.

Other selected journals from SCIRP are listed as below. Submit your manuscript to us via either submit@scirp.org or Online Submission Portal.
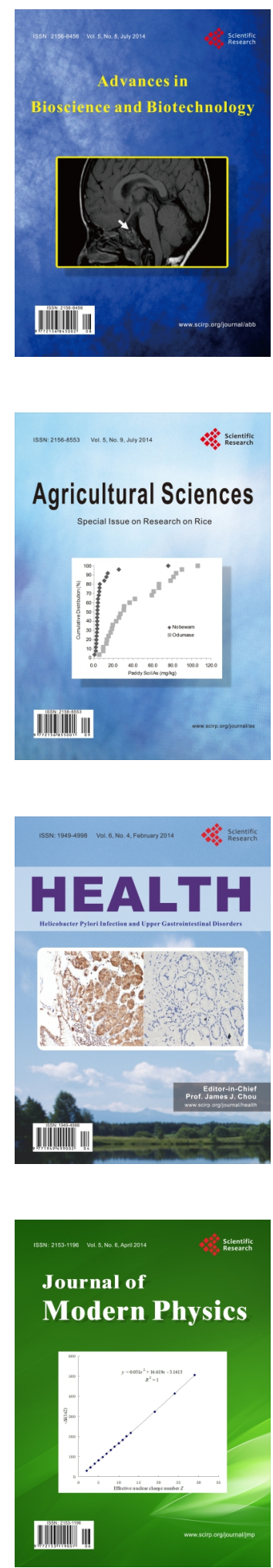
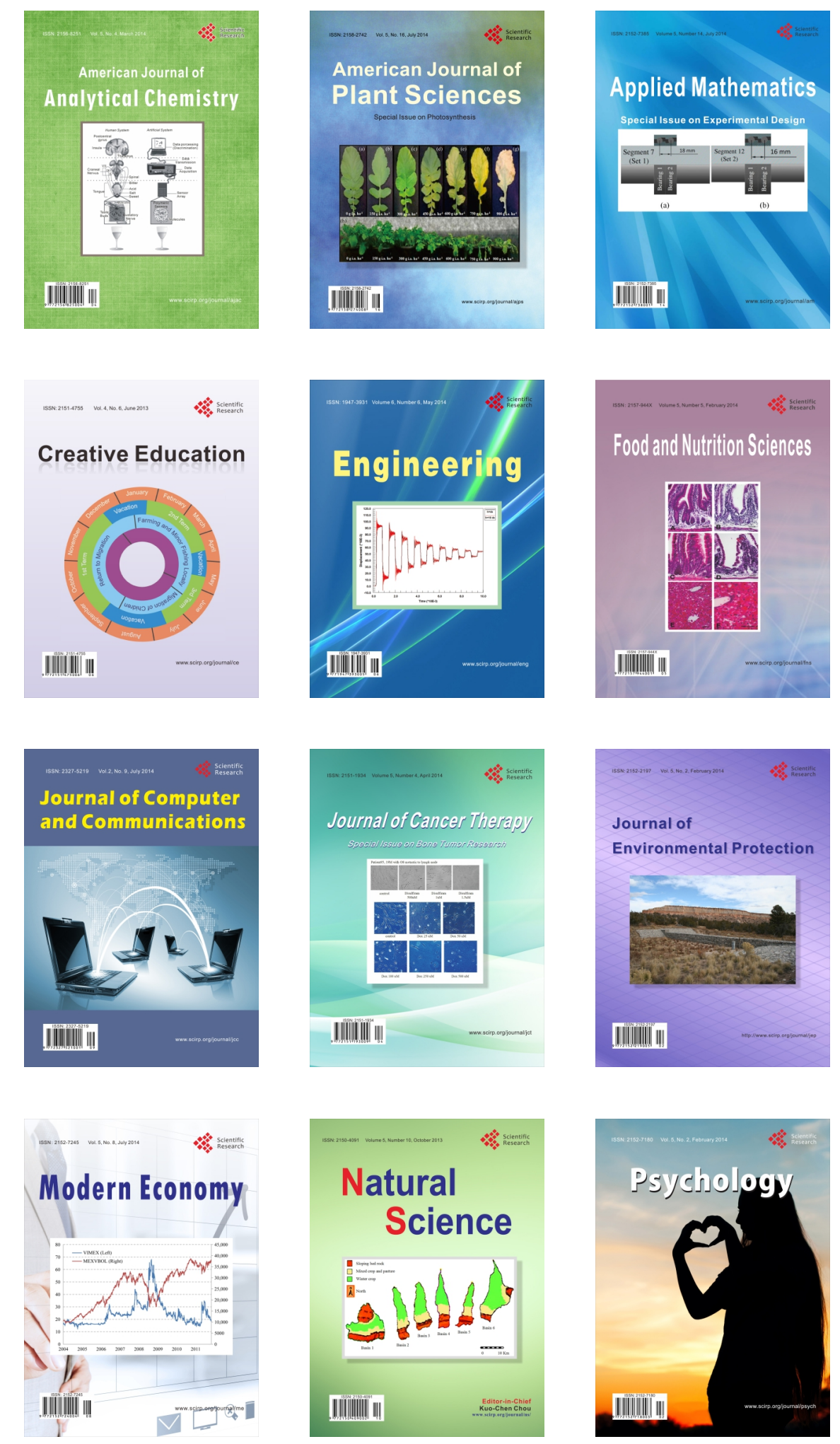